\title{
On a new approach to the analysis of variance for experiments with orthogonal block structure.
}

\section{Experiments in proper block designs}

\author{
Tadeusz Caliński, Idzi Siatkowski \\ Department of Mathematical and Statistical Methods, Poznań University of Life \\ Sciences, Wojska Polskiego 28, 60-637 Poznań, Poland, \\ e-mail: calinski@up.poznan.pl, idzi.siatkowski@up.poznan.pl
}

\begin{abstract}
SUMMARY
The main estimation and hypothesis testing results are presented for experiments conducted in proper block designs. It is shown that, under appropriate randomization, these experiments have the convenient orthogonal block structure. Because of this, the analysis of experimental data can be performed in a comparatively simple way. Certain simplifying procedures are introduced. The main advantage of the presented methodology concerns the analysis of variance and related hypothesis testing procedures. Under the adopted approach one can perform them directly, not by combining results from intra-block and inter-block analyses. Application of the theory is illustrated by three examples of real experiments in proper block designs. This is the first of a projected series of papers concerning the analysis of experiments with orthogonal block structure.
\end{abstract}

Key words: analysis of variance, estimation, hypothesis testing, orthogonal block structure, proper block designs, randomization-derived model

\section{Introduction}

The concept of orthogonal block structure, as a desirable property, was originally introduced for a wide class of designs by Nelder (1965) and then formalized by Houtman and Speed (1983). After the latter, the following definition can be adopted.

Definition 1.1 (from Section 2.2 in Houtman and Speed, 1983). An experiment is said to have the orthogonal block structure (OBS) if the covariance 
(dispersion) matrix of the random variables observed on the experimental units (plots), $\boldsymbol{y}=\left[y_{1}, y_{2}, \ldots, y_{n}\right]^{\prime}$, has a representation of the form

$$
\mathrm{D}(\boldsymbol{y})=\sigma_{1}^{2} \phi_{1}+\sigma_{2}^{2} \phi_{2}+\cdots+\sigma_{t}^{2} \phi_{t},
$$

where the $\left\{\phi_{\alpha}\right\}, \alpha=1,2, \ldots, t$, are known symmetric, idempotent and pairwise orthogonal matrices, summing to the identity matrix, the last being usually of the form $\boldsymbol{\phi}_{t}=n^{-1} \mathbf{1}_{n} \mathbf{1}_{n}^{\prime}$.

It appears that experiments having the OBS property can be analyzed in a comparatively simple way. In particular, the analysis of variance (ANOVA) can be performed directly, avoiding the classic procedure of first conducting intra-block and inter-block analyses and then combining the information obtained from them, as originally suggested by Yates $(1939,1940)$ and recently discussed by Kala (2017).

Because of this feature, it may be interesting to show the analytical advantage of experiments having the OBS property. To indicate the underlying theory and relevant methodological procedures, it will be more appealing to do this for different classes of designs separately. Thus, a set of research papers may be helpful for practical applications. The present paper, as the first of the projected series of publications, is devoted to experiments conducted in proper block designs.

Agricultural and other experiments, particularly crop variety trials, are often conducted in incomplete block designs. Of special interest are the proper block designs, i.e., those with constant block sizes. These have been intensively promoted by many researchers, who have also presented relevant methods of analyzing experiments conducted in these designs; see for example Yates $(1936,1940)$ and Rao $(1947,1956,1959)$. The considered designs, when used with appropriate randomization, induce the OBS property, as indicated in Caliński and Kageyama (2000, Lemma 3.5.1).

The purpose of the present paper is to show how the OBS property provides a possibility of performing the analysis of experimental data with a comparatively simple methodology. In Section 2 the randomization-derived mixed model, from which the described methodology follows, is introduced. The theoretical background of the derived analysis is presented in Section 3. In Section 4 some simplifications of the proposed analytical methods are suggested. In Section 5 attention is drawn to some modifications resulting from the use of estimated stratum variances. Some examples illustrating application of the derived analytical methods, ANOVA in particular, are presented in Section 6. Some concluding remarks concerning the advantage 
of the proposed new approach are given in Section 7. Finally, five appendices contain helpful derivations of the applied methods.

\section{A randomization-derived model}

Suppose that $v$ crop varieties (or other treatments) are compared in an experiment conducted in a proper block design, composed of $b$ blocks, each of $k$ experimental units (plots). Furthermore, assume that the blocks are randomized and, independently of this randomization, the plots within each block are randomized, before being assigned to the varieties according to the chosen design (as described, for example, in Caliński and Kageyama, 2000, Section 3.1.1, following Nelder, 1954, 1965).

Let the design be described by a $v \times b$ incidence matrix $\boldsymbol{N}=\left[n_{i j}\right]$, with rows corresponding to the varieties (treatments) and columns corresponding to the blocks, the element $n_{i j}$ of this matrix being positive if the variety $i$ $(=1,2, \ldots, v)$ is to be allocated in the block $j(=1,2, \ldots, b), n_{i j} \geqslant 1$ times, and being 0 otherwise.

Let the data concerning the yield (or other variable trait) observed on the plots of the experiment be written in the form of an $n \times 1$ vector $\boldsymbol{y}, n=$ $b k$, ordered as $\boldsymbol{y}=\left[\boldsymbol{y}_{1}^{\prime}, \boldsymbol{y}_{2}^{\prime}, \ldots, \boldsymbol{y}_{b}^{\prime}\right]^{\prime}$, where $\boldsymbol{y}_{j}$ is the vector of yields observed for the varieties allocated in the $j$ th block, $j=1,2, \ldots, b$. Furthermore, let the corresponding unit (plot) error and technical error variables be written in the form of $n \times 1$ vectors $\boldsymbol{\eta}$ and $\boldsymbol{e}$ respectively. Finally, let the variety (treatment) fixed effects (i.e., their expectations or true means) be written as $\boldsymbol{\tau}=\left[\tau_{1}, \tau_{2}, \ldots, \tau_{v}\right]^{\prime}$ and the block random effects as $\boldsymbol{\beta}=\left[\beta_{1}, \beta_{2}, \ldots, \beta_{b}\right]^{\prime}$.

The model derived from the adopted randomization procedures can then be written in the form

$$
\boldsymbol{y}=\boldsymbol{X}_{1} \boldsymbol{\tau}+\boldsymbol{X}_{2} \boldsymbol{\beta}+\boldsymbol{\eta}+\boldsymbol{e},
$$

with the associated design matrices $\boldsymbol{X}_{1}$ for treatments and $\boldsymbol{X}_{2}$ for blocks. This allows the expectation vector and the covariance (dispersion) matrix of $\boldsymbol{y}$ to be written as

$$
\begin{aligned}
\mathrm{E}(\boldsymbol{y}) & =\boldsymbol{X}_{1} \boldsymbol{\tau}, \\
\mathrm{D}(\boldsymbol{y}) \equiv \boldsymbol{V} & =\sigma_{1}^{2} \boldsymbol{\phi}_{1}+\sigma_{2}^{2} \boldsymbol{\phi}_{2}+\sigma_{3}^{2} \phi_{3},
\end{aligned}
$$

where the matrices

$\phi_{1}=\boldsymbol{I}_{n}-k^{-1} \boldsymbol{X}_{2} \boldsymbol{X}_{2}^{\prime}, \quad \phi_{2}=k^{-1} \boldsymbol{X}_{2} \boldsymbol{X}_{2}^{\prime}-n^{-1} \mathbf{1}_{n} \mathbf{1}_{n}^{\prime}$ and $\phi_{3}=n^{-1} \mathbf{1}_{n} \mathbf{1}_{n}^{\prime}$ 
are the pairwise orthogonal projectors on relevant strata (intra-block, interblock and total experimental area, respectively), i.e., are symmetric, idempotent and pairwise orthogonal, summing to the identity matrix, and where $\sigma_{1}^{2}, \sigma_{2}^{2}$ and $\sigma_{3}^{2}$ are the corresponding stratum variances (as in Caliński and Kageyama, 2000, Definition 3.5.1).

\section{Theoretical background of the analysis}

When analyzing data from an experiment modelled by (1), a variety trial in particular, attention is usually paid to estimates and tests concerning the parameters $\boldsymbol{\tau}=\left[\tau_{1}, \tau_{2}, \ldots, \tau_{v}\right]^{\prime}$, or rather the variety (treatment) main effects, defined as

$$
\left(\boldsymbol{I}_{v}-n^{-1} \mathbf{1}_{v} \boldsymbol{r}^{\prime}\right) \boldsymbol{\tau}=\left[\tau_{1}-\tau ., \tau_{2}-\tau ., \ldots, \tau_{v}-\tau .\right]^{\prime}, \text { where } \tau .=n^{-1} \sum_{i=1}^{v}\left(r_{i} \tau_{i}\right),
$$

and also their linear functions. In this regard, first note (referring, for instance, to Caliński and Kageyama, 2000, Section A.2) that, taking the orthogonal $\left(\boldsymbol{V}^{-1}\right.$-orthogonal) projector

$$
\boldsymbol{P}_{X_{1}\left(V^{-1}\right)}=\boldsymbol{X}_{1}\left(\boldsymbol{X}_{1}^{\prime} \boldsymbol{V}^{-1} \boldsymbol{X}_{1}\right)^{-1} \boldsymbol{X}_{1}^{\prime} \boldsymbol{V}^{-1},
$$

one can decompose the vector $\boldsymbol{y}$ in (1) into two uncorrelated parts, as

$$
\boldsymbol{y}=\boldsymbol{P}_{X_{1}\left(V^{-1}\right)} \boldsymbol{y}+\left(\boldsymbol{I}_{n}-\boldsymbol{P}_{X_{1}\left(V^{-1}\right)}\right) \boldsymbol{y} .
$$

The role of the two parts on the right in (5) will be seen in the following considerations.

Under the model (1), with properties (2) and (3), the first term of the partition in (5) provides the best linear unbiased estimator (BLUE) of $\boldsymbol{X}_{1} \boldsymbol{\tau}$ in (2), which can be expressed as

$$
\widehat{\boldsymbol{X}_{1} \boldsymbol{\tau}}=\boldsymbol{P}_{X_{1}\left(V^{-1}\right)} \boldsymbol{y}
$$

as follows from Rao (1974, Theorem 3.2). With regard to the second term in (5), it can be seen as the residual vector, giving the residual sum of squares in the form

$$
\begin{aligned}
\left\|\left(\boldsymbol{I}_{n}-\boldsymbol{P}_{X_{1}\left(V^{-1}\right)}\right) \boldsymbol{y}\right\|_{V^{-1}}^{2} & =\boldsymbol{y}^{\prime}\left(\boldsymbol{I}_{n}-\boldsymbol{P}_{X_{1}\left(V^{-1}\right)}\right)^{\prime} \boldsymbol{V}^{-1}\left(\boldsymbol{I}_{n}-\boldsymbol{P}_{X_{1}\left(V^{-1}\right)}\right) \boldsymbol{y} \\
& =\boldsymbol{y}^{\prime}\left[\boldsymbol{V}^{-1}-\boldsymbol{V}^{-1} \boldsymbol{X}_{1}\left(\boldsymbol{X}_{1}^{\prime} \boldsymbol{V}^{-1} \boldsymbol{X}_{1}\right)^{-1} \boldsymbol{X}_{1}^{\prime} \boldsymbol{V}^{-1}\right] \boldsymbol{y} \\
& =\boldsymbol{y}^{\prime} \boldsymbol{V}^{-1}\left(\boldsymbol{I}_{n}-\boldsymbol{P}_{X_{1}\left(V^{-1}\right)}\right) \boldsymbol{y},
\end{aligned}
$$


with the residual degrees of freedom (d.f.) given by

$$
\operatorname{rank}\left(\boldsymbol{V}: \boldsymbol{X}_{1}\right)-\operatorname{rank}\left(\boldsymbol{X}_{1}\right)=n-v ;
$$

see Rao (1974, Theorem 3.4) and formula (3.17) there. For convenience note that, when using the projector (4) in the considered applications, the variance $\sigma_{3}^{2}$ in the involved matrix $\boldsymbol{V}$, defined in (3), can be replaced by 1 . This is evident from formula (3.8.15) in Caliński and Kageyama (2000).

It will also be interesting to note that, as $\boldsymbol{\tau}=\boldsymbol{r}^{-\delta} \boldsymbol{X}_{1}^{\prime} \boldsymbol{X}_{1} \boldsymbol{\tau}$, the BLUE of $\boldsymbol{\tau}$ can be obtained, on account of the formulae (4) and (6), as

$$
\hat{\boldsymbol{\tau}}=\left(\boldsymbol{X}_{1}^{\prime} \boldsymbol{V}^{-1} \boldsymbol{X}_{1}\right)^{-1} \boldsymbol{X}_{1}^{\prime} \boldsymbol{V}^{-1} \boldsymbol{y} .
$$

Its covariance (dispersion) matrix then takes the form

$$
\begin{aligned}
\mathrm{D}(\hat{\boldsymbol{\tau}}) & =\left(\boldsymbol{X}_{1}^{\prime} \boldsymbol{V}^{-1} \boldsymbol{X}_{1}\right)^{-1} \boldsymbol{X}_{1}^{\prime} \boldsymbol{V}^{-1} \mathrm{D}(\boldsymbol{y}) \boldsymbol{V}^{-1} \boldsymbol{X}_{1}\left(\boldsymbol{X}_{1}^{\prime} \boldsymbol{V}^{-1} \boldsymbol{X}_{1}\right)^{-1} \\
& =\left(\boldsymbol{X}_{1}^{\prime} \boldsymbol{V}^{-1} \boldsymbol{X}_{1}\right)^{-1} .
\end{aligned}
$$

The results (7)-(9) can be checked by referring to Theorem 3.1 in Rao (1971). For this one has to show that the equality

$$
\left[\begin{array}{cc}
\boldsymbol{V} & \boldsymbol{X}_{1} \\
\boldsymbol{X}_{1}^{\prime} & \mathbf{O}
\end{array}\right]^{-}=\left[\begin{array}{rr}
\boldsymbol{V}^{-1}\left(\boldsymbol{I}_{n}-\boldsymbol{P}_{X_{1}\left(V^{-1}\right)}\right) & \boldsymbol{V}^{-1} \boldsymbol{X}_{1}\left(\boldsymbol{X}_{1}^{\prime} \boldsymbol{V}^{-1} \boldsymbol{X}_{1}\right)^{-1} \\
\left(\boldsymbol{X}_{1}^{\prime} \boldsymbol{V}^{-1} \boldsymbol{X}_{1}\right)^{-1} \boldsymbol{X}_{1}^{\prime} \boldsymbol{V}^{-1} & -\left(\boldsymbol{X}_{1}^{\prime} \boldsymbol{V}^{-1} \boldsymbol{X}_{1}\right)^{-1}
\end{array}\right]
$$

holds. In fact, it can easily be shown that the matrix on the right in (10) is a generalized inverse ( $g$-inverse) of that on the left.

With these results one can now proceed to the concept of testing the hypothesis

$$
H_{0}:\left(\boldsymbol{I}_{v}-n^{-1} \mathbf{1}_{v} \boldsymbol{r}^{\prime}\right) \boldsymbol{\tau}=\mathbf{0} .
$$

First one has to check whether the hypothesis (11) is consistent. Evidently, it is such algebraically. For its statistical consistency note that the BLUE of $\boldsymbol{\tau}_{*}=\left(\boldsymbol{I}_{v}-n^{-1} \mathbf{1}_{v} \boldsymbol{r}^{\prime}\right) \boldsymbol{\tau}$ is $\hat{\boldsymbol{\tau}}_{*}=\left(\boldsymbol{I}_{v}-n^{-1} \mathbf{1}_{v} \boldsymbol{r}^{\prime}\right) \hat{\boldsymbol{\tau}}$, with $\hat{\boldsymbol{\tau}}$ given in (8). Its dispersion matrix is, from (9), of the form

$$
\mathrm{D}\left(\hat{\boldsymbol{\tau}}_{*}\right)=\left(\boldsymbol{I}_{v}-n^{-1} \mathbf{1}_{v} \boldsymbol{r}^{\prime}\right)\left(\boldsymbol{X}_{1}^{\prime} \boldsymbol{V}^{-1} \boldsymbol{X}_{1}\right)^{-1}\left(\boldsymbol{I}_{v}-n^{-1} \boldsymbol{r} \mathbf{1}_{v}^{\prime}\right),
$$

with rank $v-1$. It appears that as a $g$-inverse of $\mathrm{D}\left(\hat{\boldsymbol{\tau}}_{*}\right)$ one can take the matrix $\boldsymbol{X}_{1}^{\prime} \boldsymbol{V}^{-1} \boldsymbol{X}_{1}$. Hence,

$$
\mathrm{D}\left(\hat{\boldsymbol{\tau}}_{*}\right)\left[\mathrm{D}\left(\hat{\boldsymbol{\tau}}_{*}\right)\right]^{-} \hat{\boldsymbol{\tau}}_{*}=\hat{\boldsymbol{\tau}}_{*},
$$


as can be shown (see Appendix 1). The equality (13) indicates that $H_{0}$ in (11) is consistent. See formula (3.2.8) in Rao (1971).

Assuming now that $\boldsymbol{y} \sim N_{n}\left(\boldsymbol{X}_{1} \boldsymbol{\tau}, \boldsymbol{V}\right)$ and, hence, that

$\hat{\boldsymbol{\tau}}_{*} \sim N_{v}\left[\boldsymbol{\tau}_{*}, \mathrm{D}\left(\hat{\boldsymbol{\tau}}_{*}\right)\right]$, where $\boldsymbol{\tau}_{*}=\left(\boldsymbol{I}_{v}-n^{-1} \mathbf{1}_{v} \boldsymbol{r}^{\prime}\right) \boldsymbol{\tau}$ and $\mathrm{D}\left(\hat{\boldsymbol{\tau}}_{*}\right)$ is as in (12), one can test the hypothesis $H_{0}$ using the statistic

$$
F=\frac{n-v}{v-1} \frac{\mathrm{SS}_{V}}{\mathrm{SS}_{R}}=\frac{n-v}{v-1} \frac{\hat{\boldsymbol{\tau}}_{*}^{\prime} \boldsymbol{X}_{1}^{\prime} \boldsymbol{V}^{-1} \boldsymbol{X}_{1} \hat{\boldsymbol{\tau}}_{*}}{\boldsymbol{y}^{\prime} \boldsymbol{V}^{-1}\left(\boldsymbol{I}_{n}-\boldsymbol{P}_{X_{1}\left(V^{-1}\right)}\right) \boldsymbol{y}},
$$

as follows from Theorem 3.2 in Rao (1971). Note, however, that the sums of squares in (14) can equivalently be written (see Appendix 2) as

$$
\begin{gathered}
\mathrm{SS}_{V}=\boldsymbol{y}^{\prime} \boldsymbol{V}^{-1} \boldsymbol{X}_{1}\left(\boldsymbol{I}_{v}-\mathbf{1}_{v} \boldsymbol{r}^{\prime} / n\right)\left(\boldsymbol{X}_{1}^{\prime} \boldsymbol{V}^{-1} \boldsymbol{X}_{1}\right)^{-1}\left(\boldsymbol{I}_{v}-\boldsymbol{r} \mathbf{1}_{v}^{\prime} / n\right) \boldsymbol{X}_{1}^{\prime} \boldsymbol{V}^{-1} \boldsymbol{y} \\
\mathrm{SS}_{R}=\boldsymbol{y}^{\prime}\left[\boldsymbol{V}^{-1}-\boldsymbol{V}^{-1} \boldsymbol{X}_{1}\left(\boldsymbol{X}_{1}^{\prime} \boldsymbol{V}^{-1} \boldsymbol{X}_{1}\right)^{-1} \boldsymbol{X}_{1}^{\prime} \boldsymbol{V}^{-1}\right] \boldsymbol{y}
\end{gathered}
$$

Referring to Theorems 9.2.1 and 9.4.1 in Rao and Mitra (1971), one can show that the quadratic forms (15) and (16) have, independently, the following $\chi^{2}$ distributions:

$$
\begin{aligned}
& \mathrm{SS}_{V} \sim \chi^{2}(v-1, \delta), \quad \text { with } \delta=\boldsymbol{\tau}_{*}^{\prime} \boldsymbol{X}_{1}^{\prime} \boldsymbol{V}^{-1} \boldsymbol{X}_{1} \boldsymbol{\tau}_{*}, \\
& \mathrm{SS}_{R} \sim \chi^{2}(n-v, 0) .
\end{aligned}
$$

Evidently, the distribution in (17) is central if the hypothesis $H_{0}$ is true, whereas that in (18) is central whether $H_{0}$ is true or not. These results imply that the statistic (14) has a noncentral $F$ distribution with $v-1$ and $n-v$ d.f., and with the noncentrality parameter $\delta$, as in (17). Thus, the distribution is central if $H_{0}$ is true.

It should be noted, however, that the above estimation and hypothesis testing procedures are applicable if the stratum variances $\sigma_{1}^{2}, \sigma_{2}^{2}$ and $\sigma_{3}^{2}$ are known. In practice they are usually unknown and have to be estimated. Hence, the problem is how to estimate them. To answer this question, it will be helpful to return to formula (7), writing it (following Caliński and Kageyama, 2000, pp. 143-144) as

$$
\begin{aligned}
& \left\|\left(\boldsymbol{I}_{n}-\boldsymbol{P}_{X_{1}\left(V^{-1}\right)}\right) \boldsymbol{y}\right\|_{V^{-1}}^{2}= \\
& =\boldsymbol{y}^{\prime}\left(\boldsymbol{I}_{n}-\boldsymbol{P}_{X_{1}\left(V^{-1}\right)}\right)^{\prime} \boldsymbol{V}^{-1}\left(\boldsymbol{I}_{n}-\boldsymbol{P}_{X_{1}\left(V^{-1}\right)}\right) \boldsymbol{y} \\
& =\sigma_{1}^{-2} \boldsymbol{y}^{\prime}\left(\boldsymbol{I}_{n}-\boldsymbol{P}_{X_{1}\left(V^{-1}\right)}\right)^{\prime} \boldsymbol{\phi}_{1}\left(\boldsymbol{I}_{n}-\boldsymbol{P}_{X_{1}\left(V^{-1}\right)}\right) \boldsymbol{y} \\
& +\sigma_{2}^{-2} \boldsymbol{y}^{\prime}\left(\boldsymbol{I}_{n}-\boldsymbol{P}_{X_{1}\left(V^{-1}\right)}\right)^{\prime} \boldsymbol{\phi}_{2}\left(\boldsymbol{I}_{n}-\boldsymbol{P}_{X_{1}\left(V^{-1}\right)}\right) \boldsymbol{y},
\end{aligned}
$$


which follows from the form of $\mathrm{D}(\boldsymbol{y}) \equiv \boldsymbol{V}$, given in (3). This form also implies, because of $\boldsymbol{\phi}_{3}=n^{-1} \mathbf{1}_{n} \mathbf{1}_{n}^{\prime}=n^{-1} \mathbf{1}_{n} \mathbf{1}_{v}^{\prime} \boldsymbol{X}_{1}^{\prime}$, that

$$
\phi_{3}\left(\boldsymbol{I}_{n}-\boldsymbol{P}_{X_{1}\left(V^{-1}\right)}\right)=\sigma_{3}^{2} \boldsymbol{\phi}_{3} \boldsymbol{V}^{-1}\left(\boldsymbol{I}_{n}-\boldsymbol{P}_{X_{1}\left(V^{-1}\right)}\right)=\mathbf{O} .
$$

Now, from (19), one can write

$$
\begin{aligned}
\mathrm{E}\left\{\left\|\left(\boldsymbol{I}_{n}-\boldsymbol{P}_{X_{1}\left(V^{-1}\right)}\right) \boldsymbol{y}\right\|_{V^{-1}}^{2}\right\}= & \sigma_{1}^{-2} \mathrm{E}\left\{\left\|\phi_{1}\left(\boldsymbol{I}_{n}-\boldsymbol{P}_{X_{1}\left(V^{-1}\right)}\right) \boldsymbol{y}\right\|^{2}\right\} \\
& +\sigma_{2}^{-2} \mathrm{E}\left\{\left\|\phi_{2}\left(\boldsymbol{I}_{n}-\boldsymbol{P}_{X_{1}\left(V^{-1}\right)}\right) \boldsymbol{y}\right\|^{2}\right\} \\
= & d_{1}^{\prime}+d_{2}^{\prime}=n-v,
\end{aligned}
$$

because, as can be shown (see again Caliński and Kageyama, 2000, p. 144),

$$
\mathrm{E}\left\{\left\|\boldsymbol{\phi}_{1}\left(\boldsymbol{I}_{n}-\boldsymbol{P}_{X_{1}\left(V^{-1}\right)}\right) \boldsymbol{y}\right\|^{2}\right\}=\sigma_{1}^{2} d_{1}^{\prime},
$$

where $d_{1}^{\prime}=\operatorname{tr}\left[\boldsymbol{\phi}_{1}\left(\boldsymbol{I}_{n}-\boldsymbol{P}_{X_{1}\left(V^{-1}\right)}\right)\right]$,

$$
\mathrm{E}\left\{\left\|\phi_{2}\left(\boldsymbol{I}_{n}-\boldsymbol{P}_{X_{1}\left(V^{-1}\right)}\right) \boldsymbol{y}\right\|^{2}\right\}=\sigma_{2}^{2} d_{2}^{\prime},
$$

where $d_{2}^{\prime}=\operatorname{tr}\left[\boldsymbol{\phi}_{2}\left(\boldsymbol{I}_{n}-\boldsymbol{P}_{X_{1}\left(V^{-1}\right)}\right)\right]$.

With these results it is natural to consider as estimators of $\sigma_{1}^{2}$ and $\sigma_{2}^{2}$ the solutions of the equations

$$
\begin{aligned}
\left\|\phi_{1}\left(\boldsymbol{I}_{n}-\boldsymbol{P}_{X_{1}\left(V^{-1}\right)}\right) \boldsymbol{y}\right\|^{2} & =\sigma_{1}^{2} d_{1}^{\prime}, \\
\left\|\phi_{2}\left(\boldsymbol{I}_{n}-\boldsymbol{P}_{X_{1}\left(V^{-1}\right)}\right) \boldsymbol{y}\right\|^{2} & =\sigma_{2}^{2} d_{2}^{\prime},
\end{aligned}
$$

respectively (as suggested by Nelder, 1968, Section 3).

The above approach, which can be considered as a generalized alternative to that introduced by Yates $(1939,1940)$, is also advocated by Houtman and Speed (1983, Sections 4.5 and 5.1). For completeness, it will be helpful to note that the equations (23) and (24), with the formulae (21) and (22), imply on account of (20) the equality

$$
\begin{aligned}
& \hat{\sigma}_{1}^{-2}\left\|\phi_{1}\left(\boldsymbol{I}_{n}-\boldsymbol{P}_{X\left(V^{-1}\right)}\right) \boldsymbol{y}\right\|^{2}+\hat{\sigma}_{2}^{-2}\left\|\boldsymbol{\phi}_{2}\left(\boldsymbol{I}_{n}-\boldsymbol{P}_{X\left(V^{-1}\right)}\right) \boldsymbol{y}\right\|^{2}= \\
& =d_{1}^{\prime}+d_{2}^{\prime}=n-v .
\end{aligned}
$$

Now, returning to (16), note that, after some algebraic transformations, it can be written equivalently as

$$
\begin{aligned}
\mathrm{SS}_{R}= & \boldsymbol{y}^{\prime}\left[\boldsymbol{I}_{n}-\boldsymbol{V}^{-1} \boldsymbol{X}_{1}\left(\boldsymbol{X}_{1}^{\prime} \boldsymbol{V}^{-1} \boldsymbol{X}_{1}\right)^{-1} \boldsymbol{X}_{1}^{\prime}\right]\left(\sigma_{1}^{-2} \phi_{1}+\sigma_{2}^{-2} \phi_{2}\right)\left[\boldsymbol{I}_{n}\right. \\
& \left.-\boldsymbol{X}_{1}\left(\boldsymbol{X}_{1}^{\prime} \boldsymbol{V}^{-1} \boldsymbol{X}_{1}\right)^{-1} \boldsymbol{X}_{1}^{\prime} \boldsymbol{V}^{-1}\right] \boldsymbol{y} \\
= & \sigma_{1}^{-2}\left\|\boldsymbol{\phi}_{1}\left(\boldsymbol{I}_{n}-\boldsymbol{P}_{X\left(V^{-1}\right)}\right) \boldsymbol{y}\right\|^{2}+\sigma_{2}^{-2}\left\|\phi_{2}\left(\boldsymbol{I}_{n}-\boldsymbol{P}_{X\left(V^{-1}\right)}\right) \boldsymbol{y}\right\|^{2} .
\end{aligned}
$$


A comparison of formulae (25) and (26) shows that if the stratum variances $\sigma_{1}^{2}$ and $\sigma_{2}^{2}$ are estimated by solutions of the equations (23) and (24), the result

$$
\begin{aligned}
\widehat{\mathrm{SS}}_{R}= & \hat{\sigma}_{1}^{-2}\left\|\boldsymbol{\phi}_{1}\left(\boldsymbol{I}_{n}-\boldsymbol{P}_{X\left(V^{-1}\right)}\right) \boldsymbol{y}\right\|^{2} \\
& +\hat{\sigma}_{2}^{-2}\left\|\boldsymbol{\phi}_{2}\left(\boldsymbol{I}_{n}-\boldsymbol{P}_{X\left(V^{-1}\right)}\right) \boldsymbol{y}\right\|^{2}=n-v
\end{aligned}
$$

then follows. On account of $(27)$ the statistic $F$ in (14) is reduced to the form

$$
\widehat{F}=\frac{n-v}{v-1} \frac{\widehat{\mathrm{SS}}_{V}}{n-v}=\frac{\widehat{\mathrm{SS}}_{V}}{v-1}
$$

where $\widehat{\mathrm{SS}}_{V}$ is as in (15) but with $\sigma_{1}^{2}$ and $\sigma_{2}^{2}$ there replaced by their estimates.

However, the $\chi^{2}$ distribution of $\mathrm{SS}_{V}$, indicated in (17), is valid only if the true stratum variances are used in the applied matrix $\boldsymbol{V}^{-1}=\sigma_{1}^{-2} \phi_{1}+$ $\sigma_{2}^{-2} \phi_{2}+\sigma_{3}^{-2} \phi_{3}$, resulting from (3). As to the component $\sigma_{3}^{-2} \phi_{3}$, it does not in fact play any role in the application of the formula (15) given for $\mathrm{SS}_{V}$ (as will be shown in the next section). Thus, when using in $\boldsymbol{V}^{-1}$ the estimates $\hat{\sigma}_{1}^{-2}$ and $\hat{\sigma}_{2}^{-2}$ obtained from (23) and (24) respectively, the $\chi^{2}$ distribution in (17) can be regarded as approximate only.

\section{Some simplifying reformulations}

As was remarked in the previous section, the component $\sigma_{3}^{-2} \phi_{3}$ in the formula $\boldsymbol{V}^{-1}=\sigma_{1}^{-2} \boldsymbol{\phi}_{1}+\sigma_{2}^{-2} \boldsymbol{\phi}_{2}+\sigma_{3}^{-2} \boldsymbol{\phi}_{3}$ seems to play no role in the formulae applicable in the considered analysis of experimental data. This suggests that some reformulation in the methodology presented in Section 3 would simplify the analysis without changing its results.

A desirable simplification can be obtained when the matrix $\boldsymbol{V}$ in the form given in (3) is replaced by the matrix $\boldsymbol{V}_{*}=\sigma_{1}^{2} \boldsymbol{\phi}_{1}+\sigma_{2}^{2}\left(\boldsymbol{I}_{n}-\boldsymbol{\phi}_{1}\right)$, i.e., when replacing the inverted matrix $\boldsymbol{V}^{-1}$ by $\boldsymbol{V}_{*}^{-1}=\sigma_{1}^{-2} \boldsymbol{\phi}_{1}+\sigma_{2}^{-2}\left(\boldsymbol{I}_{n}-\right.$ $\left.\phi_{1}\right)$. The relations between $\boldsymbol{V}$ and $\boldsymbol{V}_{*}$, and their inverses, are given by the equalities

$$
\boldsymbol{V}=\boldsymbol{V}_{*}+\left(\sigma_{3}^{2}-\sigma_{2}^{2}\right) n^{-1} \mathbf{1}_{n} \mathbf{1}_{n}^{\prime} ; \quad \boldsymbol{V}^{-1}=\boldsymbol{V}_{*}^{-1}+\left(\sigma_{3}^{-2}-\sigma_{2}^{-2}\right) n^{-1} \mathbf{1}_{n} \mathbf{1}_{n}^{\prime} .
$$

From (29) it follows (see Appendix 3) that

$$
\left(\boldsymbol{X}_{1}^{\prime} \boldsymbol{V}^{-1} \boldsymbol{X}_{1}\right)^{-1}=\left(\boldsymbol{X}_{1}^{\prime} \boldsymbol{V}_{*}^{-1} \boldsymbol{X}_{1}\right)^{-1}+\left(\sigma_{3}^{2}-\sigma_{2}^{2}\right) n^{-1} \mathbf{1}_{v} \mathbf{1}_{v}^{\prime} .
$$


Applying the equality (30), it can be shown (see again Appendix 3) that the BLUE of $\boldsymbol{\tau}_{*}=\left(\boldsymbol{I}_{v}-n^{-1} \mathbf{1}_{v} \boldsymbol{r}^{\prime}\right) \boldsymbol{\tau}$ following from (8), i.e.,

$$
\hat{\boldsymbol{\tau}}_{*}=\left(\boldsymbol{I}_{v}-n^{-1} \mathbf{1}_{v} \boldsymbol{r}^{\prime}\right) \hat{\boldsymbol{\tau}}=\left(\boldsymbol{I}_{v}-n^{-1} \mathbf{1}_{v} \boldsymbol{r}^{\prime}\right)\left(\boldsymbol{X}_{1}^{\prime} \boldsymbol{V}^{-1} \boldsymbol{X}_{1}\right)^{-1} \boldsymbol{X}_{1}^{\prime} \boldsymbol{V}^{-1} \boldsymbol{y},
$$

can equivalently be written as

$$
\hat{\boldsymbol{\tau}}_{*}=\left(\boldsymbol{I}_{v}-n^{-1} \mathbf{1}_{v} \boldsymbol{r}^{\prime}\right)\left(\boldsymbol{X}_{1}^{\prime} \boldsymbol{V}_{*}^{-1} \boldsymbol{X}_{1}\right)^{-1} \boldsymbol{X}_{1}^{\prime} \boldsymbol{V}_{*}^{-1} \boldsymbol{y}_{*},
$$

where $\boldsymbol{y}_{*}=\left(\boldsymbol{I}_{n}-n^{-1} \mathbf{1}_{n} \mathbf{1}_{n}^{\prime}\right) \boldsymbol{y}$, with $\mathrm{E}\left(\boldsymbol{y}_{*}\right)=\left(\boldsymbol{I}_{n}-n^{-1} \mathbf{1}_{n} \mathbf{1}_{n}^{\prime}\right) \boldsymbol{X}_{1} \boldsymbol{\tau}=\boldsymbol{X}_{1}\left(\boldsymbol{I}_{v}-\right.$ $\left.n^{-1} \mathbf{1}_{v} \boldsymbol{r}^{\prime}\right) \boldsymbol{\tau}=\boldsymbol{X}_{1} \boldsymbol{\tau}_{*}$ and $\mathrm{D}\left(\boldsymbol{y}_{*}\right)=\left(\boldsymbol{I}_{n}-n^{-1} \mathbf{1}_{n} \mathbf{1}_{n}^{\prime}\right) \boldsymbol{V}_{*}\left(\boldsymbol{I}_{n}-n^{-1} \mathbf{1}_{n} \mathbf{1}_{n}^{\prime}\right)$. The dispersion matrix of $\hat{\boldsymbol{\tau}}_{*}$, given in (12), can on account of (30) be presented as

$$
\mathrm{D}\left(\hat{\boldsymbol{\tau}}_{*}\right)=\left(\boldsymbol{I}_{v}-n^{-1} \mathbf{1}_{v} \boldsymbol{r}^{\prime}\right)\left(\boldsymbol{X}_{1}^{\prime} \boldsymbol{V}_{*}^{-1} \boldsymbol{X}_{1}\right)^{-1}\left(\boldsymbol{I}_{v}-n^{-1} \boldsymbol{r} \mathbf{1}_{v}^{\prime}\right) .
$$

Furthermore, the formulae for $\mathrm{SS}_{V}$ and $\mathrm{SS}_{R}$, given in (15) and (16), can equivalently be written (see Appendices 3 and 4) as

$$
\begin{aligned}
& \mathrm{SS}_{V}=\hat{\boldsymbol{\tau}}_{*}^{\prime} \boldsymbol{X}_{1}^{\prime} \boldsymbol{V}_{*}^{-1} \boldsymbol{X}_{1} \hat{\boldsymbol{\tau}}_{*}=\boldsymbol{y}_{*}^{\prime} \boldsymbol{V}_{*}^{-1} \boldsymbol{X}_{1}\left(\boldsymbol{X}_{1}^{\prime} \boldsymbol{V}_{*}^{-1} \boldsymbol{X}_{1}\right)^{-1} \boldsymbol{X}_{1}^{\prime} \boldsymbol{V}_{*}^{-1} \boldsymbol{y}_{*}, \\
& \mathrm{SS}_{R}=\boldsymbol{y}_{*}^{\prime}\left[\boldsymbol{V}_{*}^{-1}-\boldsymbol{V}_{*}^{-1} \boldsymbol{X}_{1}\left(\boldsymbol{X}_{1}^{\prime} \boldsymbol{V}_{*}^{-1} \boldsymbol{X}_{1}\right)^{-1} \boldsymbol{X}_{1}^{\prime} \boldsymbol{V}_{*}^{-1}\right] \boldsymbol{y}_{*},
\end{aligned}
$$

with $\boldsymbol{y}_{*}$ defined as in (31). The formulae (33) and (34) provide the sum

$$
\mathrm{SS}_{V}+\mathrm{SS}_{R}=\boldsymbol{y}_{*}^{\prime} \boldsymbol{V}_{*}^{-1} \boldsymbol{y}_{*}=\mathrm{SS}_{T} \quad \text { (say), }
$$

which can be called the total sum of squares. Referring again to Rao and Mitra (1971, Theorem 9.2.1), it can be shown that

$$
\mathrm{SS}_{T} \sim \chi^{2}(n-1, \delta), \quad \text { with } \delta=\boldsymbol{\tau}_{*}^{\prime} \boldsymbol{X}_{1}^{\prime} \boldsymbol{V}_{*}^{-1} \boldsymbol{X}_{1} \boldsymbol{\tau}_{*} .
$$

In fact, as can easily be shown, the form of $\delta$ in (35) is equivalent to that given in (17). These results can be summarized in the form of an analysis of variance (ANOVA) table, as presented in Table 1.

Table 1. Analysis of variance for an experiment in a proper block design

\begin{tabular}{lccc}
\hline Source of variation & Degrees of freedom & Sum of squares & Expected mean square \\
\hline Treatments & $v-1$ & $\mathrm{SS}_{V}$ & $1+\delta /(v-1)$ \\
Residuals & $n-v$ & $\mathrm{SS}_{R}$ & 1 \\
\hline Total & $n-1$ & $\mathrm{SS}_{T}$ & - \\
\hline
\end{tabular}


The presentation of ANOVA results in Table 1 corresponds well with the formula (14) for the relevant $F$ statistic.

Suppose now that after rejecting the hypothesis (11) one is interested in testing a hypothesis $H_{0, \mathrm{~L}}: \boldsymbol{U}_{\mathrm{L}}^{\prime} \boldsymbol{\tau}=\mathbf{0}$, where $\boldsymbol{U}_{\mathrm{L}}^{\prime} \mathbf{1}_{v}=\mathbf{0}$. Note that this hypothesis, concerning a set of contrasts among treatment parameters, can also be written, under $\boldsymbol{U}_{\mathrm{L}}^{\prime} \mathbf{1}_{v}=\mathbf{0}$, as

$$
H_{0, \mathrm{~L}}: \boldsymbol{U}_{\mathrm{L}}^{\prime} \boldsymbol{\tau}_{*}=\mathbf{0}, \quad \text { where } \quad \boldsymbol{\tau}_{*}=\left(\boldsymbol{I}_{v}-n^{-1} \mathbf{1}_{v} \boldsymbol{r}^{\prime}\right) \boldsymbol{\tau},
$$

which shows that $H_{0, \mathrm{~L}}$ is implied by $H_{0}$ given in (11). To find the relevant sum of squares, first note that the BLUE of $\boldsymbol{U}_{\mathrm{L}}^{\prime} \boldsymbol{\tau}_{*}$ is, on account of (31), of the form

$$
\boldsymbol{U}_{\mathrm{L}}^{\prime} \hat{\boldsymbol{\tau}}_{*}=\boldsymbol{U}_{\mathrm{L}}^{\prime} \hat{\boldsymbol{\tau}}=\boldsymbol{U}_{\mathrm{L}}^{\prime}\left(\boldsymbol{X}_{1}^{\prime} \boldsymbol{V}_{*}^{-1} \boldsymbol{X}_{1}\right)^{-1} \boldsymbol{X}_{1}^{\prime} \boldsymbol{V}_{*}^{-1} \boldsymbol{y}_{*} .
$$

Its dispersion matrix is, on account of (32), of the form

$$
\mathrm{D}\left(\boldsymbol{U}_{\mathrm{L}}^{\prime} \hat{\boldsymbol{\tau}}_{*}\right)=\boldsymbol{U}_{\mathrm{L}}^{\prime}\left(\boldsymbol{X}_{1}^{\prime} \boldsymbol{V}_{*}^{-1} \boldsymbol{X}_{1}\right)^{-1} \boldsymbol{U}_{\mathrm{L}}
$$

Note that, applying Lemma 2.2.6(c) from Rao and Mitra (1971), one can write

$$
\boldsymbol{U}_{\mathrm{L}}^{\prime}\left(\boldsymbol{X}_{1}^{\prime} \boldsymbol{V}_{*}^{-1} \boldsymbol{X}_{1}\right)^{-1} \boldsymbol{U}_{\mathrm{L}}\left[\boldsymbol{U}_{\mathrm{L}}^{\prime}\left(\boldsymbol{X}_{1}^{\prime} \boldsymbol{V}_{*}^{-1} \boldsymbol{X}_{1}\right)^{-1} \boldsymbol{U}_{\mathrm{L}}\right]^{-} \boldsymbol{U}_{\mathrm{L}}^{\prime}=\boldsymbol{U}_{\mathrm{L}}^{\prime}
$$

which, with (38), gives the equality $\mathrm{D}\left(\boldsymbol{U}_{\mathrm{L}}^{\prime} \hat{\boldsymbol{\tau}}_{*}\right)\left[\mathrm{D}\left(\boldsymbol{U}_{\mathrm{L}}^{\prime} \hat{\boldsymbol{\tau}}_{*}\right)\right]^{-} \boldsymbol{U}_{\mathrm{L}}^{\prime} \hat{\boldsymbol{\tau}}_{*}=\boldsymbol{U}_{\mathrm{L}}^{\prime} \hat{\boldsymbol{\tau}}_{*}$. This shows that the hypothesis given in (36) is consistent, both algebraically and statistically. The relevant sum of squares can then be obtained (following Theorem 3.2 of Rao, 1971) in the form

$$
\begin{aligned}
\operatorname{SS}\left(\boldsymbol{U}_{\mathrm{L}}\right) & =\hat{\boldsymbol{\tau}}_{*}^{\prime} \boldsymbol{U}_{\mathrm{L}}\left[\mathrm{D}\left(\boldsymbol{U}_{\mathrm{L}}^{\prime} \hat{\boldsymbol{\tau}}_{*}\right)\right]^{-} \boldsymbol{U}_{\mathrm{L}}^{\prime} \hat{\boldsymbol{\tau}}_{*} \\
& =\hat{\boldsymbol{\tau}}_{*}^{\prime} \boldsymbol{U}_{\mathrm{L}}\left[\boldsymbol{U}_{\mathrm{L}}^{\prime}\left(\boldsymbol{X}_{1}^{\prime} \boldsymbol{V}_{*}^{-1} \boldsymbol{X}_{1}\right)^{-1} \boldsymbol{U}_{\mathrm{L}}\right]^{-} \boldsymbol{U}_{\mathrm{L}}^{\prime} \hat{\boldsymbol{\tau}}_{*},
\end{aligned}
$$

with the d.f. equal to $\operatorname{rank}\left(\boldsymbol{U}_{\mathrm{L}}\right)$, i.e., equal to $\operatorname{rank}\left[\mathrm{D}\left(\boldsymbol{U}_{\mathrm{L}}^{\prime} \hat{\boldsymbol{\tau}}_{*}\right)\right]$. Note that $\boldsymbol{U}_{\mathrm{L}}^{\prime} \hat{\boldsymbol{\tau}}_{*}$ is given in (37), and $\left[\boldsymbol{U}_{\mathrm{L}}^{\prime}\left(\boldsymbol{X}_{1}^{\prime} \boldsymbol{V}_{*}^{-1} \boldsymbol{X}_{1}\right)^{-1} \boldsymbol{U}_{\mathrm{L}}\right]^{-}$follows from (38). Also note, referring to Lemma 2.2.6(d) in Rao and Mitra (1971), that $\boldsymbol{U}_{\mathrm{L}}\left[\boldsymbol{U}_{\mathrm{L}}^{\prime}\left(\boldsymbol{X}_{1}^{\prime} \boldsymbol{V}_{*}^{-1} \boldsymbol{X}_{1}\right)^{-1} \boldsymbol{U}_{\mathrm{L}}\right]^{-} \boldsymbol{U}_{\mathrm{L}}^{\prime}$ is invariant for any choice of the involved $g$-inverse and is of rank equal to the rank of $\boldsymbol{U}_{\mathrm{L}}$. Of course, if the columns of $\boldsymbol{U}_{\mathrm{L}}$ are linearly independent,

$$
\left[\boldsymbol{U}_{\mathrm{L}}^{\prime}\left(\boldsymbol{X}_{1}^{\prime} \boldsymbol{V}_{*}^{-1} \boldsymbol{X}_{1}\right)^{-1} \boldsymbol{U}_{\mathrm{L}}\right]^{-} \text {becomes }\left[\boldsymbol{U}_{\mathrm{L}}^{\prime}\left(\boldsymbol{X}_{1}^{\prime} \boldsymbol{V}_{*}^{-1} \boldsymbol{X}_{1}\right)^{-1} \boldsymbol{U}_{\mathrm{L}}\right]^{-1} .
$$


Now, following the assumption $\boldsymbol{y} \sim N_{n}\left(\boldsymbol{X}_{1} \boldsymbol{\tau}, \boldsymbol{V}\right)$, adopted in Section 3, one can also assume that $\boldsymbol{U}_{\mathrm{L}}^{\prime} \hat{\boldsymbol{\tau}}_{*} \sim N\left[\boldsymbol{U}_{\mathrm{L}}^{\prime} \boldsymbol{\tau}_{*}, \mathrm{D}\left(\boldsymbol{U}_{\mathrm{L}}^{\prime} \hat{\boldsymbol{\tau}}_{*}\right)\right]$. With this, applying Theorem 9.2.3 from Rao and Mitra (1971), it can be shown, using the equality (39), that

$$
\operatorname{SS}\left(\boldsymbol{U}_{\mathrm{L}}\right) \sim \chi^{2}\left[\operatorname{rank}\left(\boldsymbol{U}_{\mathrm{L}}\right), \delta_{L}\right], \quad \text { with } \quad \delta_{\mathrm{L}}=\boldsymbol{\tau}_{*}^{\prime} \boldsymbol{U}_{\mathrm{L}}\left[\mathrm{D}\left(\boldsymbol{U}_{\mathrm{L}}^{\prime} \hat{\boldsymbol{\tau}}_{*}\right)\right]^{-} \boldsymbol{U}_{\mathrm{L}}^{\prime} \boldsymbol{\tau}_{*},
$$

this distribution being central, i.e., with $\delta_{\mathrm{L}}=0$, if $H_{0, \mathrm{~L}}$ is true.

If there are several sets of contrasts for which individual hypothesis testing is of interest, then for each of them the sum of squares presented in (40) can be used accordingly. In some situations a relevant partition of the treatment (variety) sum of squares, as given in (33), may be of interest in the application of ANOVA. The question then arises, of what kind of conditions the chosen sets of contrasts have to satisfy. It can be shown (see Appendix 5) that for two such sets of contrasts, e.g. $\boldsymbol{U}_{\mathrm{A}}^{\prime} \boldsymbol{\tau}_{*}$ and $\boldsymbol{U}_{\mathrm{B}}^{\prime} \boldsymbol{\tau}_{*}$, the equality

$$
\mathrm{SS}\left(\boldsymbol{U}_{\mathrm{A}}\right)+\mathrm{SS}\left(\boldsymbol{U}_{\mathrm{B}}\right)=\mathrm{SS}_{V}
$$

holds, for any vector $\hat{\boldsymbol{\tau}}_{*}=\left(\boldsymbol{I}_{v}-n^{-1} \mathbf{1}_{v} \boldsymbol{r}^{\prime}\right) \hat{\boldsymbol{\tau}}$, if and only if

$$
\begin{aligned}
& \left(\boldsymbol{X}_{1}^{\prime} \boldsymbol{V}_{*}^{-1} \boldsymbol{X}_{1}\right)^{-1} \boldsymbol{U}_{\mathrm{A}}\left[\boldsymbol{U}_{\mathrm{A}}^{\prime}\left(\boldsymbol{X}_{1}^{\prime} \boldsymbol{V}_{*}^{-1} \boldsymbol{X}_{1}\right)^{-1} \boldsymbol{U}_{\mathrm{A}}\right]^{-} \boldsymbol{U}_{\mathrm{A}}^{\prime} \\
& +\left(\boldsymbol{X}_{1}^{\prime} \boldsymbol{V}_{*}^{-1} \boldsymbol{X}_{1}\right)^{-1} \boldsymbol{U}_{\mathrm{B}}\left[\boldsymbol{U}_{\mathrm{B}}^{\prime}\left(\boldsymbol{X}_{1}^{\prime} \boldsymbol{V}_{*}^{-1} \boldsymbol{X}_{1}\right)^{-1} \boldsymbol{U}_{\mathrm{B}}\right]^{-} \boldsymbol{U}_{\mathrm{B}}^{\prime} \\
& =\boldsymbol{I}_{v}-n^{-1} \mathbf{1}_{v} \boldsymbol{r}^{\prime} .
\end{aligned}
$$

This, in turn, implies that

$$
\boldsymbol{U}_{\mathrm{B}}^{\prime}\left(\boldsymbol{X}_{1}^{\prime} \boldsymbol{V}_{*}^{-1} \boldsymbol{X}_{1}\right)^{-1} \boldsymbol{U}_{\mathrm{A}}=\mathbf{O} .
$$

These results can be extended for any number of considered sets of contrasts used in a partition of the type (41). The condition (43) can then be written as

$$
\boldsymbol{U}_{\mathrm{L}}^{\prime}\left(\boldsymbol{X}_{1}^{\prime} \boldsymbol{V}_{*}^{-1} \boldsymbol{X}_{1}\right)^{-1} \boldsymbol{U}_{\mathrm{L}^{*}}=\mathbf{O} \quad \text { for } \quad \mathrm{L} \neq \mathrm{L}^{*} .
$$

It may be interesting to note that for some classes of designs this condition reduces to $\boldsymbol{U}_{\mathrm{L}}^{\prime} \boldsymbol{U}_{\mathrm{L}^{*}}=\mathbf{O}$. This is true, in particular, for any balanced incomplete block (BIB) design (see, for example, Definition 2.4.2 in Caliński and Kageyama, 2000). 


\section{Application with estimated stratum variances}

The hypothesis testing procedures presented in Section 4 are fully applicable if the stratum variances $\sigma_{1}^{2}$ and $\sigma_{2}^{2}$ are known. As already mentioned in Section 3, in practical applications these variances are usually unknown and have to be estimated. This can be done by solving the equations (23) and (24). However, with these estimates the residual sum of squares $\mathrm{SS}_{R}$, presented in (16) and equivalently in (34), is reduced to $n-v$, the corresponding d.f., as shown in formula (27). This leads to corresponding reduction of the $F$ statistic (14) to that presented in (28). The estimated treatment (variety) sum of squares appearing there, $\widehat{\mathrm{SS}}_{V}$, can, on account of formulae (33), (34) and their sum, be written as

$$
\widehat{\mathrm{SS}}_{V}=\boldsymbol{y}_{*}^{\prime} \hat{\boldsymbol{V}}_{*}^{-1} \boldsymbol{y}_{*}-(n-v) \equiv \widehat{\mathrm{SS}}_{T}-n+v .
$$

In the case of known (true) values of $\sigma_{1}^{2}$ and $\sigma_{2}^{2}$ the distribution of $\mathrm{SS}_{T}=$ $\boldsymbol{y}_{*}^{\prime} \boldsymbol{V}_{*}^{-1} \boldsymbol{y}_{*}$ is as given in (35). If the hypothesis $H_{0}$ given in (11) holds, then $\delta=0$ and the distribution is central. However, the indicated distribution of $\mathrm{SS}_{T}$ is fully applicable only if the true stratum variances $\sigma_{1}^{2}$ and $\sigma_{2}^{2}$ appearing in $\boldsymbol{V}_{*}^{-1}=\sigma_{1}^{-2} \boldsymbol{\phi}_{1}+\sigma_{2}^{-2}\left(\boldsymbol{I}_{n}-\boldsymbol{\phi}_{1}\right)$ are used. Because now the matrix $\boldsymbol{V}_{*}^{-1}$ is replaced by

$$
\hat{\boldsymbol{V}}_{*}^{-1}=\hat{\sigma}_{1}^{-2} \boldsymbol{\phi}_{1}+\hat{\sigma}_{2}^{-2}\left(\boldsymbol{I}_{n}-\boldsymbol{\phi}_{1}\right),
$$

the estimated total sum of squares $\widehat{\mathrm{SS}}_{T}$, appearing in (45), does not have an exact $\chi^{2}$ distribution with $n-1$ d.f., as shown in (35). That distribution can, however, be considered as an approximation of the real distribution of $\widehat{\mathrm{SS}}_{T}$. This approximation will be the closer the larger is the number $n$, i.e., the size of the experiment.

With this approximation, the estimated mean square $\widehat{\mathrm{MS}}_{V}=\widehat{\mathrm{SS}}_{V} /(v-1)$ may be treated in a practical application as having (under $H_{0}$ ) approximately the distribution of $\chi^{2}(v-1,0) /(v-1)$, as follows from the relation in (45).

Thus, referring the test statistic $(28)$ to the $\chi^{2}(v-1,0) /(v-1)$ distribution, one will obtain an approximate test of the hypothesis $H_{0}$ formulated in (11). This means that when calculating the relevant $P$ values for testing $H_{0}$, or hypotheses implied by $H_{0}$, one has to consider them as approximate. The results obtained by Volaufova (2009) seem to suggest that the above ANOVA type $F$ test approximation will in most cases provide reasonably accurate $P$ values. 
On a new approach to the analysis of experiments in proper block designs 103

\section{Examples}

The methods considered in the previous sections will now be illustrated using data from three experiments conducted in different proper block designs. The analysis concerning the first of these experiments (Example 1) illustrates the methods of obtaining the general ANOVA, as presented in Table 1, and also the partitioned ANOVA, usually of interest for factorial experiments. The analysis applied to the other two experiments (Examples 2 and 3 ) is confined to the general ANOVA. All required computations were performed with the use of $\mathrm{R}$ ( $\mathrm{R}$ Core Team, 2017).

Example 1. Ceranka and Kaczmarek (1998) analyzed data from a plant breeding field experiment with 28 barley genotypes compared in a block design based on the incidence matrix

$$
\boldsymbol{N}=\left[\boldsymbol{N}_{1}: \boldsymbol{N}_{2}\right],
$$

where

$$
\mathbf{N}_{1}=\left[\begin{array}{llllllllllllllllll}
1 & 0 & 1 & 0 & 0 & 0 & 0 & 1 & 0 & 0 & 0 & 1 & 1 & 0 & 0 & 0 & 0 & 0 \\
1 & 1 & 0 & 1 & 0 & 0 & 0 & 0 & 1 & 0 & 0 & 0 & 1 & 1 & 0 & 0 & 0 & 0 \\
0 & 1 & 1 & 0 & 1 & 0 & 0 & 0 & 0 & 1 & 0 & 0 & 0 & 1 & 1 & 0 & 0 & 0 \\
1 & 0 & 1 & 1 & 0 & 1 & 0 & 0 & 0 & 0 & 1 & 0 & 0 & 0 & 1 & 1 & 0 & 0 \\
0 & 1 & 0 & 1 & 1 & 0 & 1 & 0 & 0 & 0 & 0 & 1 & 0 & 0 & 0 & 1 & 1 & 0 \\
0 & 0 & 0 & 1 & 0 & 1 & 1 & 1 & 0 & 0 & 0 & 0 & 0 & 1 & 0 & 0 & 0 & 1 \\
0 & 0 & 1 & 0 & 0 & 0 & 1 & 1 & 1 & 0 & 1 & 0 & 0 & 0 & 0 & 0 & 1 & 0 \\
0 & 0 & 0 & 0 & 1 & 0 & 0 & 1 & 0 & 1 & 1 & 0 & 1 & 0 & 0 & 0 & 0 & 0 \\
0 & 0 & 0 & 0 & 0 & 1 & 0 & 0 & 1 & 0 & 1 & 1 & 0 & 1 & 0 & 0 & 0 & 0 \\
0 & 0 & 0 & 0 & 0 & 0 & 1 & 0 & 0 & 1 & 0 & 1 & 1 & 0 & 1 & 0 & 0 & 0 \\
0 & 0 & 0 & 0 & 0 & 0 & 0 & 1 & 0 & 0 & 0 & 1 & 0 & 1 & 1 & 0 & 1 & 0 \\
0 & 0 & 0 & 0 & 0 & 0 & 0 & 0 & 1 & 0 & 0 & 0 & 1 & 0 & 1 & 1 & 0 & 1 \\
1 & 0 & 0 & 0 & 0 & 0 & 0 & 0 & 0 & 1 & 0 & 0 & 0 & 1 & 0 & 1 & 1 & 0 \\
0 & 1 & 0 & 0 & 0 & 0 & 0 & 0 & 0 & 0 & 1 & 0 & 0 & 0 & 1 & 0 & 1 & 1 \\
0 & 0 & 1 & 0 & 0 & 0 & 0 & 0 & 0 & 0 & 0 & 1 & 0 & 0 & 0 & 1 & 0 & 1 \\
0 & 0 & 0 & 1 & 0 & 0 & 0 & 0 & 0 & 0 & 0 & 0 & 1 & 0 & 0 & 0 & 1 & 0 \\
0 & 0 & 0 & 0 & 1 & 0 & 0 & 0 & 0 & 0 & 0 & 0 & 0 & 1 & 0 & 0 & 0 & 1 \\
0 & 0 & 0 & 0 & 0 & 1 & 0 & 0 & 0 & 0 & 0 & 0 & 0 & 0 & 1 & 0 & 0 & 0 \\
0 & 0 & 0 & 0 & 0 & 0 & 1 & 0 & 0 & 0 & 0 & 0 & 0 & 0 & 0 & 1 & 0 & 0 \\
1 & 1 & 0 & 0 & 0 & 0 & 0 & 1 & 0 & 0 & 0 & 0 & 0 & 0 & 0 & 0 & 0 & 1 \\
0 & 1 & 1 & 0 & 0 & 0 & 0 & 0 & 1 & 0 & 0 & 0 & 0 & 0 & 0 & 0 & 0 & 0 \\
0 & 0 & 1 & 1 & 0 & 0 & 0 & 0 & 0 & 1 & 0 & 0 & 0 & 0 & 0 & 0 & 0 & 0 \\
0 & 0 & 0 & 1 & 1 & 0 & 0 & 0 & 0 & 0 & 1 & 0 & 0 & 0 & 0 & 0 & 0 & 0 \\
1 & 0 & 0 & 0 & 1 & 1 & 0 & 0 & 0 & 0 & 0 & 1 & 0 & 0 & 0 & 0 & 0 & 0 \\
0 & 1 & 0 & 0 & 0 & 1 & 1 & 0 & 0 & 0 & 0 & 0 & 1 & 0 & 0 & 0 & 0 & 0 \\
0 & 0 & 0 & 0 & 1 & 0 & 0 & 1 & 1 & 0 & 0 & 0 & 0 & 0 & 0 & 1 & 0 & 0 \\
0 & 0 & 0 & 0 & 0 & 1 & 0 & 0 & 1 & 1 & 0 & 0 & 0 & 0 & 0 & 0 & 1 & 0 \\
1 & 0 & 0 & 0 & 0 & 0 & 1 & 0 & 0 & 1 & 1 & 0 & 0 & 0 & 0 & 0 & 0 & 1
\end{array}\right]
$$




$\mathbf{N}_{2}=\left[\begin{array}{llllllllllllllllll}0 & 1 & 0 & 0 & 0 & 0 & 0 & 0 & 0 & 0 & 0 & 1 & 0 & 0 & 0 & 1 & 0 & 1 \\ 0 & 0 & 1 & 0 & 0 & 0 & 0 & 0 & 0 & 0 & 0 & 0 & 1 & 0 & 0 & 0 & 1 & 0 \\ 0 & 0 & 0 & 1 & 0 & 0 & 0 & 0 & 0 & 0 & 0 & 0 & 0 & 1 & 0 & 0 & 0 & 1 \\ 0 & 0 & 0 & 0 & 1 & 0 & 0 & 0 & 0 & 0 & 0 & 0 & 0 & 0 & 1 & 0 & 0 & 0 \\ 0 & 0 & 0 & 0 & 0 & 1 & 0 & 0 & 0 & 0 & 0 & 0 & 0 & 0 & 0 & 1 & 0 & 0 \\ 1 & 0 & 0 & 0 & 0 & 0 & 0 & 1 & 0 & 0 & 0 & 0 & 0 & 0 & 0 & 0 & 0 & 1 \\ 0 & 0 & 1 & 1 & 0 & 0 & 0 & 0 & 0 & 0 & 1 & 0 & 0 & 0 & 0 & 0 & 0 & 0 \\ 1 & 0 & 0 & 0 & 1 & 1 & 0 & 0 & 0 & 0 & 0 & 0 & 1 & 0 & 0 & 0 & 0 & 0 \\ 0 & 1 & 0 & 0 & 0 & 1 & 1 & 0 & 0 & 0 & 0 & 0 & 0 & 1 & 0 & 0 & 0 & 0 \\ 0 & 0 & 1 & 0 & 0 & 0 & 1 & 1 & 0 & 0 & 0 & 0 & 0 & 0 & 1 & 0 & 0 & 0 \\ 0 & 0 & 0 & 0 & 1 & 0 & 0 & 0 & 1 & 1 & 0 & 0 & 0 & 0 & 0 & 0 & 1 & 0 \\ 0 & 0 & 0 & 0 & 0 & 1 & 0 & 0 & 0 & 1 & 1 & 0 & 0 & 0 & 0 & 0 & 0 & 1 \\ 1 & 0 & 0 & 0 & 0 & 0 & 1 & 0 & 0 & 0 & 1 & 1 & 0 & 0 & 0 & 0 & 0 & 0 \\ 0 & 1 & 0 & 0 & 0 & 0 & 0 & 1 & 0 & 0 & 0 & 1 & 1 & 0 & 0 & 0 & 0 & 0 \\ 1 & 0 & 1 & 0 & 0 & 0 & 0 & 0 & 1 & 0 & 0 & 0 & 1 & 1 & 0 & 0 & 0 & 0 \\ 1 & 1 & 0 & 1 & 0 & 0 & 0 & 0 & 0 & 1 & 0 & 0 & 0 & 1 & 1 & 0 & 0 & 0 \\ 0 & 1 & 1 & 0 & 1 & 0 & 0 & 0 & 0 & 0 & 1 & 0 & 0 & 0 & 1 & 1 & 0 & 0 \\ 1 & 0 & 1 & 1 & 0 & 1 & 0 & 0 & 0 & 0 & 0 & 1 & 0 & 0 & 0 & 1 & 1 & 0 \\ 0 & 1 & 0 & 1 & 1 & 0 & 1 & 0 & 0 & 0 & 0 & 0 & 1 & 0 & 0 & 0 & 1 & 1 \\ 0 & 0 & 0 & 1 & 0 & 1 & 1 & 0 & 1 & 0 & 0 & 0 & 0 & 0 & 1 & 0 & 0 & 0 \\ 1 & 0 & 0 & 0 & 1 & 0 & 1 & 1 & 0 & 1 & 0 & 0 & 0 & 0 & 0 & 1 & 0 & 0 \\ 0 & 1 & 0 & 0 & 0 & 1 & 0 & 1 & 1 & 0 & 1 & 0 & 0 & 0 & 0 & 0 & 1 & 0 \\ 0 & 0 & 1 & 0 & 0 & 0 & 1 & 0 & 1 & 1 & 0 & 1 & 0 & 0 & 0 & 0 & 0 & 1 \\ 0 & 0 & 0 & 1 & 0 & 0 & 0 & 1 & 0 & 1 & 1 & 0 & 1 & 0 & 0 & 0 & 0 & 0 \\ 0 & 0 & 0 & 0 & 1 & 0 & 0 & 0 & 1 & 0 & 1 & 1 & 0 & 1 & 0 & 0 & 0 & 0 \\ 0 & 0 & 0 & 0 & 0 & 0 & 0 & 1 & 0 & 0 & 0 & 1 & 0 & 1 & 1 & 0 & 1 & 0 \\ 0 & 0 & 0 & 0 & 0 & 0 & 0 & 0 & 1 & 0 & 0 & 0 & 1 & 0 & 1 & 1 & 0 & 1 \\ 0 & 0 & 0 & 0 & 0 & 0 & 0 & 0 & 0 & 1 & 0 & 0 & 0 & 1 & 0 & 1 & 1 & 0\end{array}\right]$

This represents a BIB design with parameters $v=28, r=9, b=36, k=$ $7, \lambda=2$ (as recorded at No. 76 in Table 8.2 of Caliński and Kageyama, 2003). The design can be presented more transparently as in Table 2.

As follows from the considerations in Sections 8.2 of Caliński and Kageyama (2003), the design used here is a binary proper and equireplicate design with the parameters given above, and with the efficiency factor $\varepsilon_{1}=$ $\lambda v /(r k)=8 / 9=0.8889$ of multiplicity $\rho_{1}=v-1=27$.

In this example the role of the $v$ treatments (genotypes) is played by 28 progenies (three-line hybrids) obtained from the triallel crossing between 7 lines (first group of parental lines, P), 2 testers (second group of parental lines, Q) and 2 varieties (third group of parental lines, R) of barley. (For details of the triallel crossing system see Ceranka and Kaczmarek, 1998, and the references given there.) Thus, the analyzed data can well be considered as resulting from a factorial experiment with three factors, P, Q and R, applied at 7,2 and 2 levels respectively. The labels of the progenies are ordered lexicographically with respect to the levels of the factors $\mathrm{P}, \mathrm{Q}$ and $\mathrm{R}$, in the sense that those labeled 1,2,3,4 correspond to the first level of $\mathrm{P}$, the first two of them to the first level of $\mathrm{Q}$, and the very first of 
On a new approach to the analysis of experiments in proper block designs 105

them to the first level of R, etc. As usual, it is assumed here that the compared progenies were assigned to the experimental plots subject to the randomization of blocks and plots within the blocks, in accordance with the rule described in Section 2.

Table 2. The BIB design used in Example 1 (before randomization)

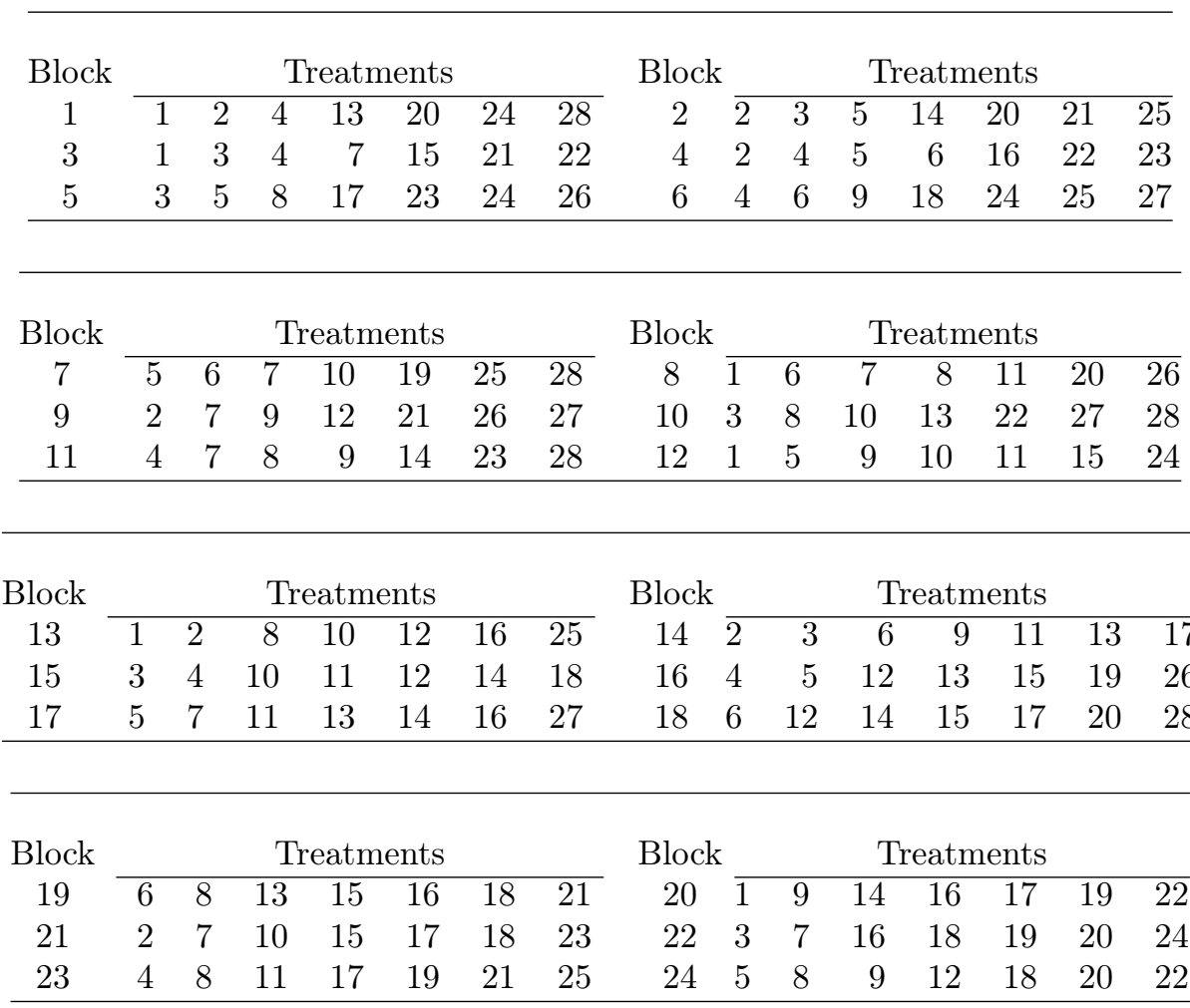

\begin{tabular}{|c|c|c|c|c|c|c|c|c|c|c|c|c|c|c|c|}
\hline \multirow{2}{*}{$\begin{array}{c}\text { Block } \\
25\end{array}$} & \multicolumn{7}{|c|}{ Treatments } & \multirow{2}{*}{$\begin{array}{c}\text { Block } \\
26\end{array}$} & \multicolumn{7}{|c|}{ Treatments } \\
\hline & 9 & 10 & 13 & 19 & 20 & 21 & 23 & & 6 & 10 & 14 & 21 & 22 & 24 & 26 \\
\hline 27 & 11 & 15 & 20 & 22 & 23 & 25 & 27 & 28 & 11 & 12 & 16 & 21 & 23 & 24 & 28 \\
\hline 29 & 7 & 12 & 13 & 17 & 22 & 24 & 25 & 30 & 1 & 13 & 14 & 18 & 23 & 25 & 26 \\
\hline
\end{tabular}

\begin{tabular}{|c|c|c|c|c|c|c|c|c|c|c|c|c|c|c|c|}
\hline \multirow{2}{*}{$\begin{array}{c}\text { Block } \\
31\end{array}$} & \multicolumn{7}{|c|}{ Treatments } & \multicolumn{3}{|c|}{ Block } & \multicolumn{5}{|c|}{ Treatments } \\
\hline & 2 & 8 & 14 & 15 & 19 & 24 & 27 & 32 & 3 & 9 & 15 & 16 & 25 & 26 & 28 \\
\hline 33 & 4 & 10 & 16 & 17 & 20 & 26 & 27 & 34 & 1 & 5 & 17 & 18 & 21 & 27 & 28 \\
\hline 35 & 2 & 11 & 18 & 19 & 22 & 26 & 28 & 36 & 1 & 3 & 6 & 12 & 19 & 23 & 27 \\
\hline
\end{tabular}


Table 3. Experimental observations of the grain yield of the barley progenies analyzed in Example 1

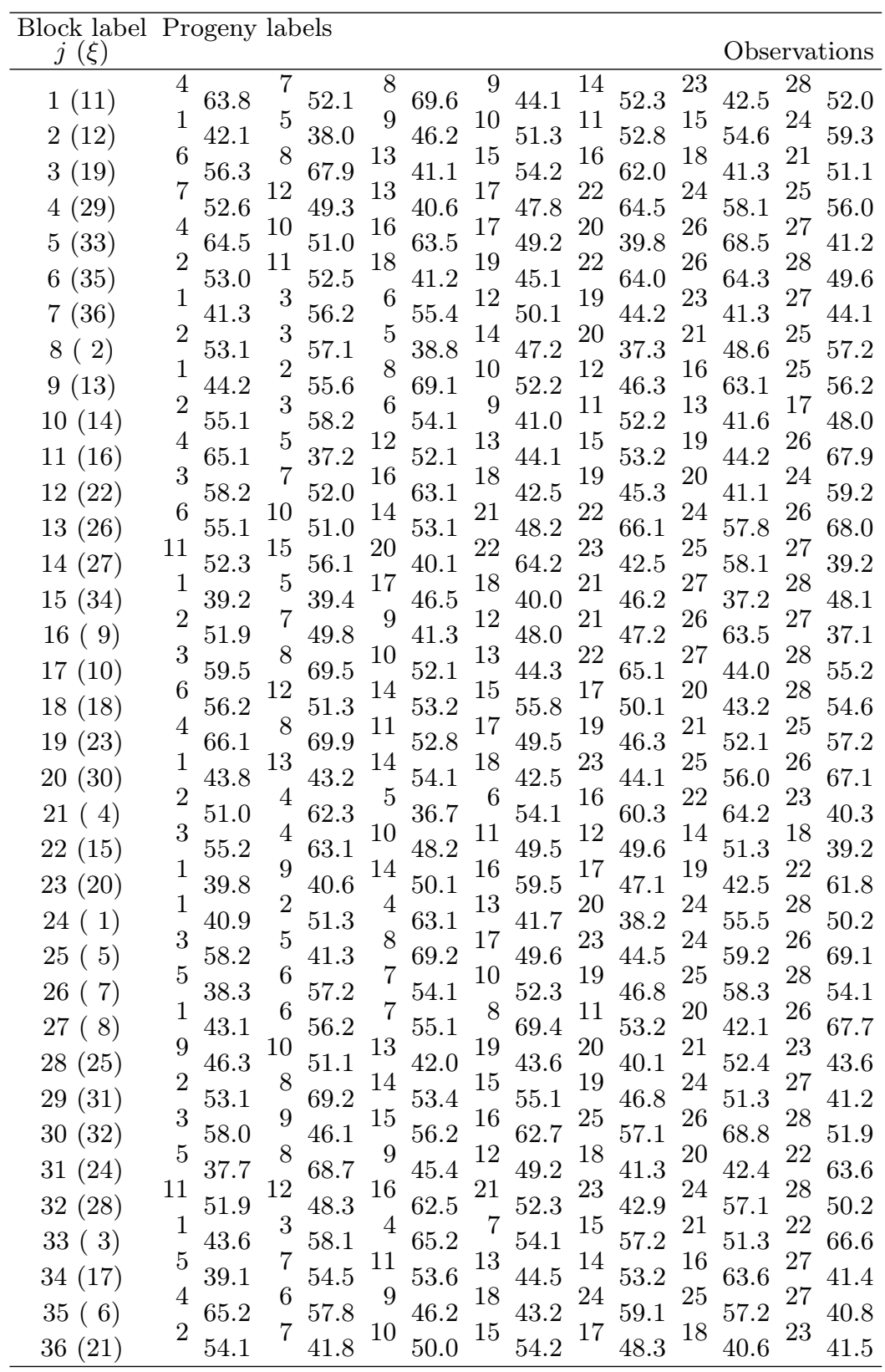


The plant trait observed on the experimental units (plots), and analyzed for this example, is the grain yield. The individual plot observations are given in Table 3. Its rows are ordered according to the actual order of blocks (labeled $j$ ) in the field layout of the experiment, $\xi$ denoting the original label of the block before randomization. The progenies are ordered as in the columns of the incidence matrix $\boldsymbol{N}$ given above.

When analyzing these data, the researcher (a plant breeder) would be interested in estimating and testing several sets of treatment parametric functions, which can be defined as follows (according to the approach of Ceranka and Kaczmarek, 1995, 1998):

$$
\begin{aligned}
\boldsymbol{g}^{\mathrm{P}} & =\left[g_{1}^{\mathrm{P}}, g_{2}^{\mathrm{P}}, \ldots, g_{7}^{\mathrm{P}}\right]^{\prime}=\left[\left(\boldsymbol{I}_{7}-\frac{1}{7} \mathbf{1}_{7} \mathbf{1}_{7}^{\prime}\right) \otimes \frac{1}{2} \mathbf{1}_{2}^{\prime} \otimes \frac{1}{2} \mathbf{1}_{2}^{\prime}\right] \boldsymbol{\tau}_{*}=\boldsymbol{U}_{\mathrm{P}}^{\prime} \boldsymbol{\tau}_{*}, \\
\boldsymbol{g}^{\mathrm{Q}} & =\left[g_{1}^{\mathrm{Q}}, g_{2}^{\mathrm{Q}}\right]^{\prime}=\left[\frac{1}{7} \mathbf{1}_{7}^{\prime} \otimes\left(\boldsymbol{I}_{2}-\frac{1}{2} \mathbf{1}_{2} \mathbf{1}_{2}^{\prime}\right) \otimes \frac{1}{2} \mathbf{1}_{2}^{\prime}\right] \boldsymbol{\tau}_{*}=\boldsymbol{U}_{\mathrm{Q}}^{\prime} \boldsymbol{\tau}_{*}, \\
\boldsymbol{g}^{\mathrm{R}} & =\left[g_{1}^{\mathrm{R}}, g_{2}^{\mathrm{R}}\right]^{\prime}=\left[\frac{1}{7} \mathbf{1}_{7}^{\prime} \otimes \frac{1}{2} \mathbf{1}_{2}^{\prime} \otimes\left(\boldsymbol{I}_{2}-\frac{1}{2} \mathbf{1}_{2} \mathbf{1}_{2}^{\prime}\right)\right] \boldsymbol{\tau}_{*}=\boldsymbol{U}_{\mathrm{R}}^{\prime} \boldsymbol{\tau}_{*}, \\
\boldsymbol{s}^{\mathrm{PQ}} & =\left[s_{11}^{\mathrm{PQ}}, s_{12}^{\mathrm{PQ}}, \ldots, s_{72}^{\mathrm{PQ}}\right]^{\prime} \\
& =\left[\left(\boldsymbol{I}_{7}-\frac{1}{7} \mathbf{1}_{7} \mathbf{1}_{7}^{\prime}\right) \otimes\left(\boldsymbol{I}_{2}-\frac{1}{2} \mathbf{1}_{2} \mathbf{1}_{2}^{\prime}\right) \otimes \frac{1}{2} \mathbf{1}_{2}^{\prime}\right] \boldsymbol{\tau}_{*}=\boldsymbol{U}_{\mathrm{PQ}}^{\prime} \boldsymbol{\tau}_{*}, \\
\boldsymbol{s}^{\mathrm{PR}} & =\left[s_{11}^{\mathrm{PR}}, s_{12}^{\mathrm{PR}}, \ldots, s_{72}^{\mathrm{PR}}\right]^{\prime} \\
& =\left[\left(\boldsymbol{I}_{7}-\frac{1}{7} \mathbf{1}_{7} \mathbf{1}_{7}^{\prime}\right) \otimes \frac{1}{2} \mathbf{1}_{2}^{\prime} \otimes\left(\boldsymbol{I}_{2}-\frac{1}{2} \mathbf{1}_{2} \mathbf{1}_{2}^{\prime}\right)\right] \boldsymbol{\tau}_{*}=\boldsymbol{U}_{\mathrm{PR}}^{\prime} \boldsymbol{\tau}_{*}, \\
\boldsymbol{s}^{\mathrm{QR}} & =\left[s_{11}^{\mathrm{QR}}, s_{12}^{\mathrm{QR}}, s_{21}^{\mathrm{QR}}, s_{22}^{\mathrm{QR}}\right]^{\prime} \\
& =\left[\frac{1}{7} \mathbf{1}_{7}^{\prime} \otimes\left(\boldsymbol{I}_{2}-\frac{1}{2} \mathbf{1}_{2} \mathbf{1}_{2}^{\prime}\right) \otimes\left(\boldsymbol{I}_{2}-\frac{1}{2} \mathbf{1}_{2} \mathbf{1}_{2}^{\prime}\right)\right] \boldsymbol{\tau}_{*}=\boldsymbol{U}_{\mathrm{QR}}^{\prime} \boldsymbol{\tau}_{*}, \\
\boldsymbol{s}^{\mathrm{PQR}} & =\left[s_{111}^{\mathrm{PQR}}, s_{112}^{\mathrm{PQR}}, \ldots, s_{722}^{\mathrm{PQR}}\right]^{\prime} \\
& =\left[\left(\boldsymbol{I}_{7}-\frac{1}{7} \mathbf{1}_{7} \mathbf{1}_{7}^{\prime}\right) \otimes\left(\boldsymbol{I}_{2}-\frac{1}{2} \mathbf{1}_{2} \mathbf{1}_{2}^{\prime}\right) \otimes\left(\boldsymbol{I}_{2}-\frac{1}{2} \mathbf{1}_{2} \mathbf{1}_{2}^{\prime}\right)\right] \boldsymbol{\tau}_{*}=\boldsymbol{U}_{\mathrm{PQR}}^{\prime} \boldsymbol{\tau}_{*},
\end{aligned}
$$

where $\boldsymbol{g}$ stands for general effects and $s$ for specific effects of the relevant parents, their pairs or triplets. Note that all of these functions are contrasts of treatment parameters, related to a complete set of basic contrasts of the design (see Definition 3.4.1 in Caliński and Kageyama, 2000). For any set of these contrasts, say $\boldsymbol{U}_{\mathrm{L}}$, the BLUE is obtainable according to formula (37), and the relevant $\operatorname{SS}\left(\boldsymbol{U}_{\mathrm{L}}\right)$ follows from (40). It may be helpful to note 
that for this example (for a BIB design) these formulae can be simplified, due to the relation

$$
\boldsymbol{U}_{\mathrm{L}}^{\prime}\left(\boldsymbol{X}_{1}^{\prime} \boldsymbol{V}_{*}^{-1} \boldsymbol{X}_{1}\right)^{-1}=\left[\varepsilon_{1} \sigma_{1}^{-2}+\left(1-\varepsilon_{1}\right) \sigma_{2}^{-2}\right]^{-1} r^{-1} \boldsymbol{U}_{\mathrm{L}}^{\prime} .
$$

It may also be interesting to note that when considering all seven sets of contrasts jointly, i.e., the set $\boldsymbol{U}^{\prime} \boldsymbol{\tau}_{*}$, where

$$
\boldsymbol{U}=\left[\boldsymbol{U}_{\mathrm{P}}: \boldsymbol{U}_{\mathrm{Q}}: \boldsymbol{U}_{\mathrm{R}}: \boldsymbol{U}_{\mathrm{PQ}}: \boldsymbol{U}_{\mathrm{PR}}: \boldsymbol{U}_{\mathrm{QR}}: \boldsymbol{U}_{\mathrm{PQR}}\right]
$$

the equality

$$
\begin{aligned}
\mathrm{SS}(\boldsymbol{U})= & \mathrm{SS}\left(\boldsymbol{U}_{\mathrm{P}}\right)+\mathrm{SS}\left(\boldsymbol{U}_{\mathrm{Q}}\right)+\mathrm{SS}\left(\boldsymbol{U}_{\mathrm{R}}\right)+\mathrm{SS}\left(\boldsymbol{U}_{\mathrm{PQ}}\right) \\
& +\mathrm{SS}\left(\boldsymbol{U}_{\mathrm{PR}}\right)+\mathrm{SS}\left(\boldsymbol{U}_{\mathrm{QR}}\right)+\mathrm{SS}\left(\boldsymbol{U}_{\mathrm{PQR}}\right)
\end{aligned}
$$

holds. This can easily be checked, noting that (for any experiment in a BIB design) $\boldsymbol{U}_{\mathrm{L}}^{\prime} \boldsymbol{U}_{\mathrm{L}^{*}}=\mathbf{0}$ for $\mathrm{L} \neq \mathrm{L}^{*}$ (with $\mathrm{L}, \mathrm{L}^{*}$ for $\mathrm{P}, \mathrm{Q}, \mathrm{R}, \mathrm{PQ}, \mathrm{PR}, \mathrm{QR}$ and $\mathrm{PQR}$ ), and hence,

$$
\begin{aligned}
\boldsymbol{U}\left(\boldsymbol{U}^{\prime} \boldsymbol{U}\right)^{-} \boldsymbol{U}^{\prime}= & \boldsymbol{U}_{\mathrm{P}}\left(\boldsymbol{U}_{\mathrm{P}}^{\prime} \boldsymbol{U}_{\mathrm{P}}\right)^{-} \boldsymbol{U}_{\mathrm{P}}^{\prime}+\boldsymbol{U}_{\mathrm{Q}}\left(\boldsymbol{U}_{\mathrm{Q}}^{\prime} \boldsymbol{U}_{\mathrm{Q}}\right)^{-} \boldsymbol{U}_{\mathrm{Q}}^{\prime}+\boldsymbol{U}_{\mathrm{R}}\left(\boldsymbol{U}_{\mathrm{R}}^{\prime} \boldsymbol{U}_{\mathrm{R}}\right)^{-} \boldsymbol{U}_{\mathrm{R}}^{\prime} \\
& +\boldsymbol{U}_{\mathrm{PQ}}\left(\boldsymbol{U}_{\mathrm{PQ}}^{\prime} \boldsymbol{U}_{\mathrm{PQ}}\right)^{-} \boldsymbol{U}_{\mathrm{PQ}}^{\prime}+\boldsymbol{U}_{\mathrm{PR}}\left(\boldsymbol{U}_{\mathrm{PR}}^{\prime} \boldsymbol{U}_{\mathrm{PR}}\right)^{-} \boldsymbol{U}_{\mathrm{PR}}^{\prime} \\
& +\boldsymbol{U}_{\mathrm{QR}}\left(\boldsymbol{U}_{\mathrm{QR}}^{\prime} \boldsymbol{U}_{\mathrm{QR}}\right)^{-} \boldsymbol{U}_{\mathrm{QR}}^{\prime}+\boldsymbol{U}_{\mathrm{PQR}}\left(\boldsymbol{U}_{\mathrm{PQR}}^{\prime} \boldsymbol{U}_{\mathrm{PQR}}\right)^{-} \boldsymbol{U}_{\mathrm{PQR}}^{\prime} \\
= & \boldsymbol{I}_{28}-28^{-1} \mathbf{1}_{28} \mathbf{1}_{28}^{\prime} .
\end{aligned}
$$

These comments correspond to the results presented in Section 4, particularly those in formulae (40)-(44), reduced considerably for BIB designs. This can be seen when comparing the formula (48) above with that in (42). It may also be helpful to note, referring to (47) and (48), that formula (40) can be reduced to $\operatorname{SS}\left(\boldsymbol{U}_{\mathrm{L}}\right)=\left[\varepsilon_{1} \sigma_{1}^{-2}+\left(1-\varepsilon_{1}\right) \sigma_{2}^{-2}\right] r \hat{\boldsymbol{\tau}}_{*}^{\prime} \boldsymbol{U}_{\mathrm{L}}\left(\boldsymbol{U}_{\mathrm{L}}^{\prime} \boldsymbol{U}_{\mathrm{L}}\right)^{-} \boldsymbol{U}_{\mathrm{L}}^{\prime} \hat{\boldsymbol{\tau}}_{*}$, and that the equality

$$
\begin{aligned}
\operatorname{SS}(\boldsymbol{U}) & =\left[\varepsilon_{1} \sigma_{1}^{-2}+\left(1-\varepsilon_{1}\right) \sigma_{2}^{-2}\right] r \hat{\boldsymbol{\tau}}_{*}^{\prime}\left(\boldsymbol{I}_{v}-v^{-1} \mathbf{1}_{v} \mathbf{1}_{v}^{\prime}\right) \hat{\boldsymbol{\tau}}_{*} \\
& =\left[\varepsilon_{1} \sigma_{1}^{-2}+\left(1-\varepsilon_{1}\right) \sigma_{2}^{-2}\right] r \hat{\boldsymbol{\tau}}_{*}^{\prime} \hat{\boldsymbol{\tau}}_{*}=\mathrm{SS}_{V}
\end{aligned}
$$

holds for the present example, as it does for any experiment in a BIB design.

Now it will be possible to present the relevant ANOVA, in its general form (as in Table 1) and in a more detailed form, corresponding to the sets of contrasts presented above. Before proceeding to this, however, one has to estimate the unavailable stratum variances $\sigma_{1}^{2}$ and $\sigma_{2}^{2}$ appearing in the matrix $\boldsymbol{V}_{*}^{-1}=\sigma_{1}^{-2} \boldsymbol{\phi}_{1}+\sigma_{2}^{-2}\left(\boldsymbol{I}_{n}-\boldsymbol{\phi}_{1}\right)$. Applying a relevant iterative 
On a new approach to the analysis of experiments in proper block designs 109

procedure (using R) to solve the equations (23) and (24), the estimates $\hat{\sigma}_{1}^{2}=1.86957$ and $\hat{\sigma}_{2}^{2}=12.43493$ were obtained after 6 iteration cycles. With these estimates, the following results concerning the ANOVA were obtained.

Table 4. Analysis of variance for an experiment in a proper block (here BIB) design - Example 1

\begin{tabular}{lccc}
\hline Source of variation & Degrees of freedom & Sum of squares & Mean square \\
\hline Treatments & 27 & 8612.792 & 318.992 \\
Residuals & 224 & 224 & 1 \\
\hline Total & 251 & 8836.792 & - \\
\hline
\end{tabular}

Table 5. Analysis of variance for the sets of contrasts considered in Example 1

\begin{tabular}{crrrrr}
\hline Source & d.f. & Sum of squares & Mean square & $\hat{F}$ & $P$ value \\
\hline Treatments & 27 & 8612.792 & 318.992 & 318.992 & $<0.0001$ \\
P & 6 & 1545.604 & 257.601 & 257.601 & $<0.0001$ \\
Q & 1 & 133.608 & 133.608 & 133.608 & $<0.0001$ \\
R & 1 & 1972.722 & 1972.722 & 1972.722 & $<0.0001$ \\
PQ & 6 & 3259.716 & 543.286 & 543.286 & $<0.0001$ \\
PR & 6 & 1570.013 & 261.669 & 261.669 & $<0.0001$ \\
QR & 1 & 28.393 & 28.393 & 28.393 & $<0.0001$ \\
PQR & 6 & 102.736 & 17.123 & 17.123 & $<0.0001$ \\
Residuals & 224 & 224 & 1 & & \\
\hline Total & 251 & 8836.792 &
\end{tabular}

The results presented in Tables 4 and 5 were obtained with the use of the empirical estimates (i.e., based on $\hat{\sigma}_{1}^{2}$ and $\hat{\sigma}_{2}^{2}$ )

$$
\begin{aligned}
& \tilde{\tau}=[42.188,54.200,57.350,64.287, \quad 38.515, \quad 55.508, \quad 51.602, \\
& 68.602,44.356,50.836,52.198,49.587,42.352,52.153 \text {, } \\
& 54.949,62.326,48.743,41.716,44.927,40.296,50.263 \text {, } \\
& 64.623,42.664,57.190,56.595,67.010,40.722, \quad 51.653]^{\prime}
\end{aligned}
$$

and

$$
\begin{aligned}
& \tilde{\boldsymbol{\tau}}_{*}=[-9.505, \quad 2.507, \quad 5.656, \quad 12.594,-13.178, \quad 3.814,-0.091 \text {, }
\end{aligned}
$$

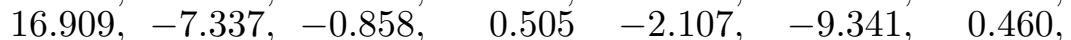

$$
\begin{aligned}
& 3.256, \quad 10.632,-2.950,-9.977, \quad-6.767,-11.398,-1.430 \text {, } \\
& 12.929,-9.030, \quad 5.497, \quad 4.902, \quad 15.317,-10.971,-0.040]^{\prime} \text {, }
\end{aligned}
$$


the former obtainable by formula (8), the latter either from the relation $\tilde{\boldsymbol{\tau}}_{*}=\left(\boldsymbol{I}_{v}-n^{-1} \mathbf{1}_{v} \boldsymbol{r}^{\prime}\right) \tilde{\boldsymbol{\tau}}$, or directly by formula (31).

The sums of squares in Table 4 were obtained by formulae (33) and (34), using the simplification for $\mathrm{SS}_{V}$ shown in (49). Not surprisingly, when $\boldsymbol{V}_{*}^{-1}$ in (34) is replaced by $\hat{\boldsymbol{V}}_{*}^{-1}$ as given in (46), the residual sum of squares $\mathrm{SS}_{R}$ is reduced to its d.f., as follows from formula (27). The sums of squares for the chosen sets of contrasts, presented in Table 5, were obtained in accordance with formula (40), which for this example (of a BIB design) is simplified with the use of the relation given in (47).

The indicated simplifications are applicable to any BIB design and, more generally, to any block design belonging to the class of efficiency-balanced (EB) designs, in the terminology of Williams (1975) and Puri and Nigam (1975a, 1975b), i.e., satisfying the condition

$$
\boldsymbol{C}_{1}=\boldsymbol{X}_{1}^{\prime} \phi_{1} \boldsymbol{X}_{1}=\varepsilon_{1}\left(\boldsymbol{r}^{\delta}-n^{-1} \boldsymbol{r} \boldsymbol{r}^{\prime}\right) .
$$

where $\varepsilon_{1}$ is the relevant efficiency factor. For more on this, see for example Caliński and Kageyama (2000, Section 4.4).

Example 2. Ceranka $(1975,1983)$ analyzed data from a plant breeding field experiment with 25 breeding strains and 2 standard varieties of sunflower compared in a block design based on the incidence matrix $\boldsymbol{N}^{*}$ of the type

$$
N^{*}=\left[\begin{array}{c}
N \\
\mathbf{1}_{s} \mathbf{1}_{b}^{\prime}
\end{array}\right]
$$

with

$$
\boldsymbol{N}=\left[\boldsymbol{N}_{1}: \boldsymbol{N}_{2}: \boldsymbol{N}_{3}: \boldsymbol{N}_{4}: \boldsymbol{N}_{5}: \boldsymbol{N}_{6}\right],
$$

where

$$
\begin{gathered}
\boldsymbol{N}_{1}^{\prime}=\left[\begin{array}{lllllllllllllllllllllllll}
1 & 1 & 1 & 1 & 1 & 0 & 0 & 0 & 0 & 0 & 0 & 0 & 0 & 0 & 0 & 0 & 0 & 0 & 0 & 0 & 0 & 0 & 0 & 0 & 0 \\
0 & 0 & 0 & 0 & 0 & 1 & 1 & 1 & 1 & 1 & 0 & 0 & 0 & 0 & 0 & 0 & 0 & 0 & 0 & 0 & 0 & 0 & 0 & 0 & 0 \\
0 & 0 & 0 & 0 & 0 & 0 & 0 & 0 & 0 & 0 & 1 & 1 & 1 & 1 & 1 & 0 & 0 & 0 & 0 & 0 & 0 & 0 & 0 & 0 & 0 \\
0 & 0 & 0 & 0 & 0 & 0 & 0 & 0 & 0 & 0 & 0 & 0 & 0 & 0 & 0 & 1 & 1 & 1 & 1 & 1 & 0 & 0 & 0 & 0 & 0 \\
0 & 0 & 0 & 0 & 0 & 0 & 0 & 0 & 0 & 0 & 0 & 0 & 0 & 0 & 0 & 0 & 0 & 0 & 0 & 0 & 1 & 1 & 1 & 1 & 1
\end{array}\right], \\
\boldsymbol{N}_{2}^{\prime}=\left[\begin{array}{llllllllllllllllllllllllll}
1 & 0 & 0 & 0 & 0 & 1 & 0 & 0 & 0 & 0 & 1 & 0 & 0 & 0 & 0 & 1 & 0 & 0 & 0 & 0 & 1 & 0 & 0 & 0 & 0 \\
0 & 1 & 0 & 0 & 0 & 0 & 1 & 0 & 0 & 0 & 0 & 1 & 0 & 0 & 0 & 0 & 1 & 0 & 0 & 0 & 0 & 1 & 0 & 0 & 0 \\
0 & 0 & 1 & 0 & 0 & 0 & 0 & 1 & 0 & 0 & 0 & 0 & 1 & 0 & 0 & 0 & 0 & 1 & 0 & 0 & 0 & 0 & 1 & 0 & 0 \\
0 & 0 & 0 & 1 & 0 & 0 & 0 & 0 & 1 & 0 & 0 & 0 & 0 & 1 & 0 & 0 & 0 & 0 & 1 & 0 & 0 & 0 & 0 & 1 & 0 \\
0 & 0 & 0 & 0 & 1 & 0 & 0 & 0 & 0 & 1 & 0 & 0 & 0 & 0 & 1 & 0 & 0 & 0 & 0 & 1 & 0 & 0 & 0 & 0 & 1
\end{array}\right],
\end{gathered}
$$




$$
\begin{aligned}
& \boldsymbol{N}_{3}^{\prime}=\left[\begin{array}{lllllllllllllllllllllllll}
1 & 0 & 0 & 0 & 0 & 0 & 1 & 0 & 0 & 0 & 0 & 0 & 1 & 0 & 0 & 0 & 0 & 0 & 1 & 0 & 0 & 0 & 0 & 0 & 1 \\
0 & 1 & 0 & 0 & 0 & 0 & 0 & 1 & 0 & 0 & 0 & 0 & 0 & 1 & 0 & 0 & 0 & 0 & 0 & 1 & 1 & 0 & 0 & 0 & 0 \\
0 & 0 & 1 & 0 & 0 & 0 & 0 & 0 & 1 & 0 & 0 & 0 & 0 & 0 & 1 & 1 & 0 & 0 & 0 & 0 & 0 & 1 & 0 & 0 & 0 \\
0 & 0 & 0 & 1 & 0 & 0 & 0 & 0 & 0 & 1 & 1 & 0 & 0 & 0 & 0 & 0 & 1 & 0 & 0 & 0 & 0 & 0 & 1 & 0 & 0 \\
0 & 0 & 0 & 0 & 1 & 1 & 0 & 0 & 0 & 0 & 0 & 1 & 0 & 0 & 0 & 0 & 0 & 1 & 0 & 0 & 0 & 0 & 0 & 1 & 0
\end{array}\right], \\
& \boldsymbol{N}_{4}^{\prime}=\left[\begin{array}{lllllllllllllllllllllllll}
1 & 0 & 0 & 0 & 0 & 0 & 0 & 1 & 0 & 0 & 0 & 0 & 0 & 0 & 1 & 0 & 1 & 0 & 0 & 0 & 0 & 0 & 0 & 1 & 0 \\
0 & 1 & 0 & 0 & 0 & 0 & 0 & 0 & 1 & 0 & 1 & 0 & 0 & 0 & 0 & 0 & 0 & 1 & 0 & 0 & 0 & 0 & 0 & 0 & 1 \\
0 & 0 & 1 & 0 & 0 & 0 & 0 & 0 & 0 & 1 & 0 & 1 & 0 & 0 & 0 & 0 & 0 & 0 & 1 & 0 & 1 & 0 & 0 & 0 & 0 \\
0 & 0 & 0 & 1 & 0 & 1 & 0 & 0 & 0 & 0 & 0 & 0 & 1 & 0 & 0 & 0 & 0 & 0 & 0 & 1 & 0 & 1 & 0 & 0 & 0 \\
0 & 0 & 0 & 0 & 1 & 0 & 1 & 0 & 0 & 0 & 0 & 0 & 0 & 1 & 0 & 1 & 0 & 0 & 0 & 0 & 0 & 0 & 1 & 0 & 0
\end{array}\right], \\
& \boldsymbol{N}_{5}^{\prime}=\left[\begin{array}{lllllllllllllllllllllllll}
1 & 0 & 0 & 0 & 0 & 0 & 0 & 0 & 1 & 0 & 0 & 1 & 0 & 0 & 0 & 0 & 0 & 0 & 0 & 1 & 0 & 0 & 1 & 0 & 0 \\
0 & 1 & 0 & 0 & 0 & 0 & 0 & 0 & 0 & 1 & 0 & 0 & 1 & 0 & 0 & 1 & 0 & 0 & 0 & 0 & 0 & 0 & 0 & 1 & 0 \\
0 & 0 & 1 & 0 & 0 & 1 & 0 & 0 & 0 & 0 & 0 & 0 & 0 & 1 & 0 & 0 & 1 & 0 & 0 & 0 & 0 & 0 & 0 & 0 & 1 \\
0 & 0 & 0 & 1 & 0 & 0 & 1 & 0 & 0 & 0 & 0 & 0 & 0 & 0 & 1 & 0 & 0 & 1 & 0 & 0 & 1 & 0 & 0 & 0 & 0 \\
0 & 0 & 0 & 0 & 1 & 0 & 0 & 1 & 0 & 0 & 1 & 0 & 0 & 0 & 0 & 0 & 0 & 0 & 1 & 0 & 0 & 1 & 0 & 0 & 0
\end{array}\right], \\
& \boldsymbol{N}_{6}^{\prime}=\left[\begin{array}{lllllllllllllllllllllllll}
1 & 0 & 0 & 0 & 0 & 0 & 0 & 0 & 0 & 1 & 0 & 0 & 0 & 1 & 0 & 0 & 0 & 1 & 0 & 0 & 0 & 1 & 0 & 0 & 0 \\
0 & 1 & 0 & 0 & 0 & 1 & 0 & 0 & 0 & 0 & 0 & 0 & 0 & 0 & 1 & 0 & 0 & 0 & 1 & 0 & 0 & 0 & 1 & 0 & 0 \\
0 & 0 & 1 & 0 & 0 & 0 & 1 & 0 & 0 & 0 & 1 & 0 & 0 & 0 & 0 & 0 & 0 & 0 & 0 & 1 & 0 & 0 & 0 & 1 & 0 \\
0 & 0 & 0 & 1 & 0 & 0 & 0 & 1 & 0 & 0 & 0 & 1 & 0 & 0 & 0 & 1 & 0 & 0 & 0 & 0 & 0 & 0 & 0 & 0 & 1 \\
0 & 0 & 0 & 0 & 1 & 0 & 0 & 0 & 1 & 0 & 0 & 0 & 1 & 0 & 0 & 0 & 1 & 0 & 0 & 0 & 1 & 0 & 0 & 0 & 0
\end{array}\right],
\end{aligned}
$$

and with

$$
\mathbf{1}_{s} \mathbf{1}_{b}^{\prime}=\left[\mathbf{1}_{2} \mathbf{1}_{5}^{\prime}: \mathbf{1}_{2} \mathbf{1}_{5}^{\prime}: \mathbf{1}_{2} \mathbf{1}_{5}^{\prime}: \mathbf{1}_{2} \mathbf{1}_{5}^{\prime}: \mathbf{1}_{2} \mathbf{1}_{5}^{\prime}: \mathbf{1}_{2} \mathbf{1}_{5}^{\prime}\right]
$$

It can be seen that the $25 \times 30$ incidence matrix $\boldsymbol{N}$ of the basic design represents a BIB design, with $v=25$ treatments (here breeding strains) replicated $r=6$ times, every two of the treatments concurring in exactly $\lambda=1$ block and each of the $b=30$ blocks being of size $k=5$.

In addition, it may be noted that the $2 \times 30$ incidence matrix $\mathbf{1}_{s} \mathbf{1}_{b}^{\prime}$ of the supplementary treatments represents a randomized complete block design, with $s=2$ treatments (here standard varieties) replicated $b=30$ times.

Thus, on account of Definition 4.4.1 in Caliński and Kageyama (2000), the resulting design given by the $27 \times 30$ incidence matrix $\boldsymbol{N}^{*}$ (see above) is a $\left(\rho_{0}^{*} ; \rho_{1}^{*} ; 0\right)$-EB design with parameters $v^{*}=v+s=27, b^{*}=b=30, \boldsymbol{r}^{*}=$ $\left[r \mathbf{1}_{v}^{\prime}, b \mathbf{1}_{s}^{\prime}\right]^{\prime}=\left[6 \mathbf{1}_{25}^{\prime}, 30 \mathbf{1}_{2}^{\prime}\right]^{\prime}, k^{*}=k+s=7$, and the efficiency factors $\varepsilon_{0}^{*}=1$, $\varepsilon_{1}^{*}=1-(r-\lambda) /[r(k+s)]=1-5 / 42=37 / 42(=0.880952)$, of multiplicities $\rho_{0}^{*}=s=2, \rho_{1}^{*}=v-1=24$. 
To complete the description of the design and its use in the analyzed experiment, it will be assumed that the randomization of blocks and of plots within the blocks has been implemented according to the procedure described in Section 2. This assumption means that the order in which the columns of the matrix $\boldsymbol{N}^{*}$ are assigned to the real blocks of plots, formed in the experimental field, has been chosen at random, and that for each block the order in which the treatments indicated by 1's in the assigned column of $\boldsymbol{N}^{*}$ are then assigned to the plots of the block has also been chosen at random.

The plant trait observed on the experimental units (plots), and taken here for analysis, is the average diameter of the capitulum (head) in centimeters. The individual plot observations are presented and analyzed in Caliński and Kageyama (2003, Example 7.3.22). The analysis applied there is based on the classic approach of first performing the intra-block and the inter-block analyses, and then combining their results. Here the direct ANOVA results, obtained by the methods described above (Sections 3 and 4), are given in Table 6 .

Table 6. Analysis of variance for an experiment in a proper block design Example 2

\begin{tabular}{lcclcc}
\hline $\begin{array}{l}\text { Source of } \\
\text { variation }\end{array}$ & $\begin{array}{c}\text { Degrees of } \\
\text { freedom }\end{array}$ & $\begin{array}{l}\text { Sum of } \\
\text { squares }\end{array}$ & $\begin{array}{l}\text { Mean } \\
\text { square }\end{array}$ & $\hat{F}$ & $P$ value \\
\hline Treatments & 26 & 87.86414 & 3.37939 & 3.37939 & $<0.0001$ \\
Residuals & 183 & 183 & 1 & - & - \\
\hline Total & 209 & 270.8641 & - & - & - \\
\hline
\end{tabular}

Similarly as in Example 1, the results presented in Table 6 were obtained using the formulae (33) and (34), but with the stratum variances $\sigma_{1}^{2}$ and $\sigma_{2}^{2}$ (appearing in the matrix $V_{*}$ ) replaced by their estimates, $\hat{\sigma}_{1}^{2}$ and $\hat{\sigma}_{2}^{2}$, obtained by solution of the equations (23) and (24). These estimates, $\hat{\sigma}_{1}^{2}=0.91893$ and $\hat{\sigma}_{2}^{2}=36.61797$ (obtained here, with the use of R, after 5 iteration cycles) coincide exactly with those obtained in the analysis presented in Caliński and Kageyama (2003, p.121). Also the empirical estimate of $\boldsymbol{\tau}$ obtained here,

$$
\begin{aligned}
& \tilde{\tau}=[15.701,15.800, \quad 15.053,15.695,16.388, \\
& 15.571,15.449, \quad 15.730,16.003,15.164 \text {, } \\
& 16.301,15.458,15.531,15.751,15.700 \text {, } \\
& 15.836,15.314,16.577,15.582,17.492 \text {, } \\
& 14.749,15.309,14.633,14.555,14.408 \text {, } \\
& 14.690,15.780]^{\prime} \text {, }
\end{aligned}
$$


is exactly the same as that given there. From this, or directly with the use of (31),

$$
\begin{aligned}
& \tilde{\boldsymbol{\tau}}_{*}=\left[\begin{array}{lllll}
0.212, & 0.311, & -0.435, & 0.206, & 0.900
\end{array}\right. \\
& 0.082,-0.039, \quad 0.241, \quad 0.515,-0.325 \text {, }
\end{aligned}
$$

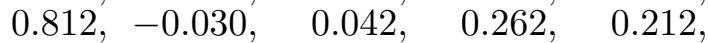

$$
\begin{aligned}
& 0.347,-0.174, \quad 1.088, \quad 0.093, \quad 2.003 \text {, } \\
& -0.740,-0.179,-0.855,-0.933,-1.081 \text {, } \\
& -0.799, \quad 0.291]^{\prime} \text {, }
\end{aligned}
$$

which by formula (33) gives $\widehat{\mathrm{SS}}_{V}=\tilde{\boldsymbol{\tau}}_{*}^{\prime} \boldsymbol{X}_{1}^{\prime} \hat{\boldsymbol{V}}_{*}^{-1} \boldsymbol{X}_{1} \tilde{\boldsymbol{\tau}}_{*}$.

Of course, with the replacement of $\sigma_{1}^{2}$ and $\sigma_{2}^{2}$ by their estimates, the sums of squares indicated in Table 6 are such that the test statistic (14) is reduced to (28). Its exact distribution under $H_{0}$ is not known, but it can be approximated by the distribution of $\chi^{2}(v-1) /(v-1)$, as indicated in Section 5. From this, the corresponding $P$ value is obtained, as given in the last column of the table.

Comparing the results summarized in Table 6 with those presented in Example 7.3.22 of Caliński and Kageyama (2003), it may be noted that in the latter only the intra-block and inter-block ANOVA tables are given. This shows the advantage of the present approach.

Example 3. Ceranka, Mejza and Wiśniewski (1979) analyzed data from a plant breeding field experiment with 12 sunflower strains compared in a block design based on the incidence matrix

$$
\boldsymbol{N}=\left[\begin{array}{llllllllllllllllll}
1 & 0 & 0 & 0 & 0 & 0 & 0 & 0 & 0 & 1 & 0 & 0 & 0 & 0 & 0 & 0 & 0 & 0 \\
0 & 1 & 0 & 0 & 0 & 0 & 0 & 0 & 0 & 0 & 1 & 0 & 0 & 0 & 0 & 0 & 0 & 0 \\
0 & 0 & 1 & 0 & 0 & 0 & 0 & 0 & 0 & 0 & 0 & 1 & 0 & 0 & 0 & 0 & 0 & 0 \\
0 & 0 & 0 & 1 & 0 & 0 & 0 & 0 & 0 & 0 & 0 & 0 & 1 & 0 & 0 & 0 & 0 & 0 \\
0 & 0 & 0 & 0 & 1 & 0 & 0 & 0 & 0 & 0 & 0 & 0 & 0 & 1 & 0 & 0 & 0 & 0 \\
0 & 0 & 0 & 0 & 0 & 1 & 0 & 0 & 0 & 0 & 0 & 0 & 0 & 0 & 1 & 0 & 0 & 0 \\
0 & 0 & 0 & 0 & 0 & 0 & 1 & 0 & 0 & 0 & 0 & 0 & 0 & 0 & 0 & 1 & 0 & 0 \\
0 & 0 & 0 & 0 & 0 & 0 & 0 & 1 & 0 & 0 & 0 & 0 & 0 & 0 & 0 & 0 & 1 & 0 \\
0 & 0 & 0 & 0 & 0 & 0 & 0 & 0 & 1 & 0 & 0 & 0 & 0 & 0 & 0 & 0 & 0 & 1 \\
2 & 2 & 2 & 0 & 0 & 0 & 0 & 0 & 0 & 2 & 2 & 2 & 0 & 0 & 0 & 0 & 0 & 0 \\
0 & 0 & 0 & 2 & 2 & 2 & 0 & 0 & 0 & 0 & 0 & 0 & 2 & 2 & 2 & 0 & 0 & 0 \\
0 & 0 & 0 & 0 & 0 & 0 & 2 & 2 & 2 & 0 & 0 & 0 & 0 & 0 & 0 & 2 & 2 & 2
\end{array}\right] .
$$

Evidently, this matrix represents a nonbinary, proper, nonequireplicate and disconnected block design, with $v_{1}=9$ treatments (here new strains) replicated $r_{1}=2$ times and $v_{2}=3$ treatments (standard strains) replicated $r_{2}=12$ times, each of the $b=18$ blocks being of size $k=3$. It is assumed 
that the randomization of blocks and of plots within the blocks has been implemented according to the procedure described in Section 2. The plant trait observed on the experimental units was again (as in Example 2) the average head diameter in centimeters. The individual plot observations are as follows (ordered as in $\boldsymbol{N}$ ):

\begin{tabular}{ccccccccc} 
Block & Strain & Observ. & \multicolumn{3}{c}{ Block } & Strain & Observ. & \multicolumn{3}{c}{ Block } & Strain & Observ. \\
1 & 1 & 12.3 & 7 & 7 & 18.4 & 13 & 4 & 18.5 \\
1 & 10 & 14.5 & 7 & 12 & 15.5 & 13 & 11 & 18.8 \\
1 & 10 & 15.0 & 7 & 12 & 15.8 & 13 & 11 & 18.7 \\
2 & 2 & 14.5 & 8 & 8 & 12.5 & 14 & 5 & 13.0 \\
2 & 10 & 15.0 & 8 & 12 & 16.0 & 14 & 11 & 19.2 \\
2 & 10 & 14.8 & 8 & 12 & 16.1 & 14 & 11 & 19.0 \\
3 & 3 & 16.4 & 9 & 9 & 19.5 & 15 & 6 & 19.0 \\
3 & 10 & 15.1 & 9 & 12 & 15.8 & 15 & 11 & 18.0 \\
3 & 10 & 15.2 & 9 & 12 & 16.0 & 15 & 11 & 18.3 \\
4 & 4 & 18.4 & 10 & 1 & 12.0 & 16 & 7 & 19.5 \\
4 & 11 & 18.8 & 10 & 10 & 15.1 & 16 & 12 & 14.9 \\
4 & 11 & 18.5 & 10 & 10 & 15.3 & 16 & 12 & 15.2 \\
5 & 5 & 14.5 & 11 & 2 & 13.5 & 17 & 8 & 12.0 \\
5 & 11 & 18.8 & 11 & 10 & 14.8 & 17 & 12 & 15.0 \\
5 & 11 & 18.7 & 11 & 10 & 15.0 & 17 & 12 & 15.5 \\
6 & 6 & 19.6 & 12 & 3 & 17.4 & 18 & 9 & 19.0 \\
6 & 11 & 19.0 & 12 & 10 & 14.5 & 18 & 12 & 15.6 \\
6 & 11 & 18.9 & 12 & 10 & 14.9 & 18 & 12 & 15.9
\end{tabular}

Because the design of this experiment does not belong to any specified class of block designs, it may be interesting to see its layout. This is presented in Table 7 .

Table 7. The proper block design used in Example 3 (before randomization)

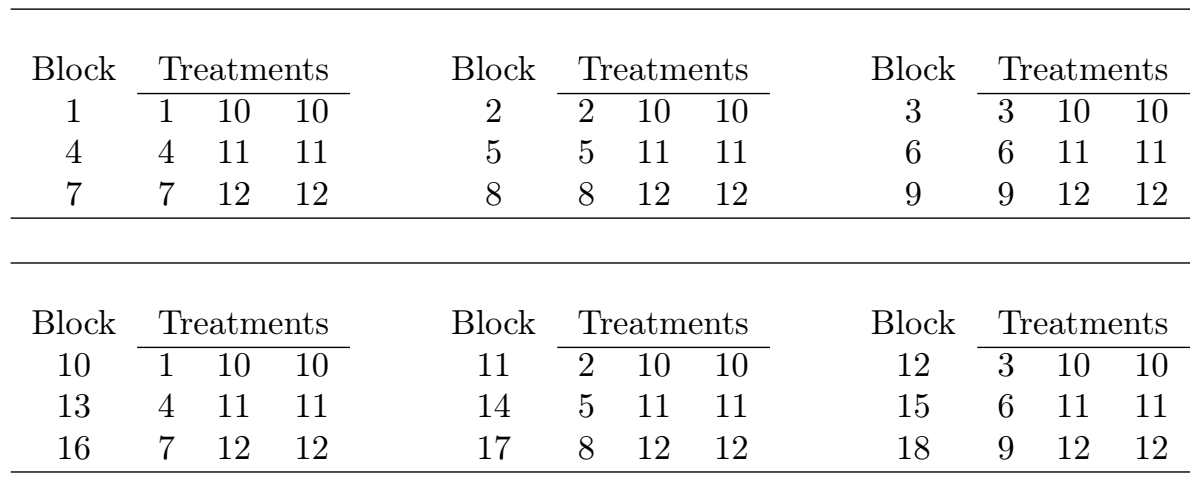

The data from the experiment considered here were analyzed in Caliński and Kageyama (2000, Example 3.8.1) following the classic approach, where intra-block and inter-block analyses are first performed, and then the results 
are combined. Here, as in the previous two examples, a direct analysis is of interest. The results of the direct ANOVA obtained by the methods described in Sections 3 and 4 are presented in Table 8.

Table 8. Analysis of variance for an experiment in a proper block design -

$$
\text { Example } 3
$$

\begin{tabular}{lccccc}
\hline $\begin{array}{l}\text { Source of } \\
\text { variation }\end{array}$ & $\begin{array}{c}\text { Degrees of } \\
\text { freedom }\end{array}$ & $\begin{array}{c}\text { Sum of } \\
\text { squares }\end{array}$ & $\begin{array}{c}\text { Mean } \\
\text { square }\end{array}$ & $\hat{F}$ & $P$ value \\
\hline Treatments & 11 & 1440.293 & 130.9358 & 130.9358 & $<0.0001$ \\
Residuals & 42 & 42 & 1 & - & - \\
\hline Total & 53 & 1482.293 & - & - & - \\
\hline
\end{tabular}

Again, the results given in Table 8 were obtained using formulae (33) and (34), but with the stratum variances $\sigma_{1}^{2}$ and $\sigma_{2}^{2}$ replaced by their estimates $\hat{\sigma}_{1}^{2}$ and $\hat{\sigma}_{2}^{2}$, obtainable as in the previous examples. Here they were obtained (at the 5th iteration cycle) as $\hat{\sigma}_{1}^{2}=0.14878$ and $\hat{\sigma}_{2}^{2}=0.19454$, which coincide with those obtained in Caliński and Kageyama (2000, p. 174).

Finally, as in the previous examples, in drawing inferences from the ANOVA results (Table 8) the empirical estimates of the parameter vectors $\boldsymbol{\tau}$ and $\boldsymbol{\tau}_{*}=\left(\boldsymbol{I}_{v}-n^{-1} \mathbf{1}_{v} \boldsymbol{r}^{\prime}\right) \boldsymbol{\tau}$ are of interest. These are obtained here as

$$
\begin{aligned}
& \tilde{\boldsymbol{\tau}}=[12.143,14.006,16.901,18.454,13.716,19.330 \text {, } \\
& 18.994,12.243,19.213,14.933,18.725,15.608]^{\prime}
\end{aligned}
$$

and

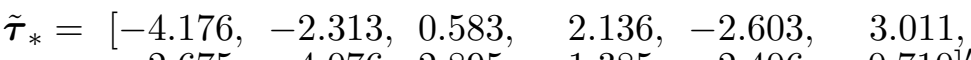

$$
\begin{aligned}
& 2.675,-4.076,2.895,-1.385, \quad 2.406,-0.710]^{\prime} \text {. }
\end{aligned}
$$

As indicated in Example 2, the latter is used in obtaining the estimated treatment sum of squares $\widehat{\mathrm{SS}_{V}}$. Also note that in all of these three examples the estimated residual sum of squares, $\widehat{\mathrm{SS}_{R}}$, is equal to the corresponding d.f., in accordance with formula (27). 


\section{Concluding remarks}

The present work was stimulated by a result obtained during the preparation of a paper by Caliński et al. (2017). It concerns the residual sum of squares, $\mathrm{SS}_{R}$, in the ANOVA applied to data from an experiment with the OBS property. It was found that when the unknown stratum variances are replaced by their estimates obtained from the estimation procedure suggested by Nelder (1968), the $\mathrm{SS}_{R}$ is reduced to its d.f., i.e., its expectation. This result is obtainable due to a proposed new approach to the analysis of experimental data.

In this paper the new approach is applied to proper block designs. The indicated result follows from the use, in the estimation and hypothesis testing procedures, of the covariance (dispersion) matrix (3) not in the form

$$
\boldsymbol{V}=\sigma_{1}^{2}\left[\boldsymbol{\phi}_{1}+\left(\sigma_{2}^{2} / \sigma_{1}^{2}\right) \boldsymbol{\phi}_{2}+\left(\sigma_{3}^{2} / \sigma_{1}^{2}\right) \boldsymbol{\phi}_{3}\right]=\sigma_{1}^{2} \boldsymbol{F} \quad \text { (say) }
$$

usually applied in the literature (as recalled recently by Kala, 2017), but in its original form $\boldsymbol{V}=\sigma_{1}^{2} \phi_{1}+\sigma_{2}^{2} \phi_{2}+\sigma_{3}^{2} \phi_{3}$, which ensures that $\mathrm{E}\left(\mathrm{SS}_{R}\right)=$ $n-v$, as follows from (18). As a consequence of this application, the test statistic (14) is reduced to the form (28), i.e., to the estimated treatment mean square. This can be seen as an advantage for the approximation of the relevant distribution.

Another feature of the proposed approach concerns simplification of the analytical procedures, as presented in Section 4. One of the resulting advantages is the reduction of the number of stratum variances involved from three to two, i.e., to $\sigma_{1}^{2}$ and $\sigma_{2}^{2}$ only. This greatly simplifies the computations.

However, as can be seen from the analyzed examples, the main advantage of the proposed approach is the fact that the ANOVA results are obtainable directly, not by first performing intra-block and inter-block analyses and then combining their results.

Finally, it is expected that this paper will be followed by others, concerning different classes of designs inducing the OBS property.

\section{Acknowledgements}

In the course of preparing the manuscript, discussions with colleagues have been appreciated. The authors thank the two anonymous referees for their encouraging comments and suggestions. 


\section{REFERENCES}

Caliński T., Czajka S., Kaczmarek Z., Krajewski P., Pilarczyk W., Siatkowski I., Siatkowski M. (2017): On a mixed model analysis of multi-environment variety trials: a reconsideration of the one-stage and the two-stage models and analyses. Statistical Paper 58: 433-465. DOI 10.1007/s00362-015-0706-y.

Caliński T., Kageyama S. (2000): Block Designs: A Randomization Approach, Vol. I: Analysis. Lecture Notes in Statistics 150, Springer, New York.

Caliński T., Kageyama S. (2003): Block Designs: A Randomization Approach, Vol. II: Design. Lecture Notes in Statistics 170, Springer, New York.

Ceranka B. (1975): Affine resolvable incomplete block designs. Zastosowania Matematyki - Applicationes Mathematicae 14: 565-572.

Ceranka B. (1983): Planning of experiments in $C$-designs. Scientific Dissertations 136, Annals of Poznań Agricultural University, Poland.

Ceranka B., Kaczmarek Z. (1995): Estimation of parameters in factorial triallel analysis for BIB design - the mixed model. In: C. P. Kitsos and W. G. Müller (eds.), Moda 4 - Advances in Model-Oriented Data Analysis. Physica-Verlag, Heidelberg, 151-156.

Ceranka B., Kaczmarek Z. (1998): Testing genetic parameters in the mixed model of triallel analysis. In: A. C. Atkinson, L. Pronzato and H. P. Wynn (eds.), Moda 5 - Advances in Model-Oriented Data Analysis and Experimental Design. Physica-Verlag, Heidelberg, 177-185.

Ceranka B., Mejza S., Wiśniewski P. (1979): Analysis of block designs with recovery of inter-block information. Roczniki Akademii Rolniczej w Poznaniu, Algorytmy Biometryczne i Statystyczne 8: 3-27 (in Polish).

Houtman A.M., Speed T.P. (1983): Balance in designed experiments with orthogonal block structure. Annals of Statistics 11: 1069-1085.

Kala R. (2017): A new look at combining information in experiments with orthogonal block structure. Matrices, Statistics and Big Data: Proceedings of the IWMS-2016 (in press).

Mardia K.V., Kent J.T., Bibby J.M. (1979): Multivariate Analysis. Academic Press, London.

Nelder J.A. (1954): The interpretation of negative components of variance. Biometrika 41: 544-548.

Nelder J.A. (1965): The analysis of randomized experiments with orthogonal block structure. Proceedings of the Royal Society of London, Series A 283: 147178.

Nelder J.A. (1968): The combination of information in generally balanced designs. Journal of the Royal Statistical Society, Series B 30: 303-311.

Puri P.D., Nigam A.K. (1975a): On patterns of efficiency balanced designs. Journal of the Royal Statistical Society, Series B 37: 457-458. 
Puri P.D., Nigam A.K. (1975b): A note on efficiency balanced designs. Sankhy-a, Series B 37: 457-460.

Rao C.R. (1947): General methods of analysis for incomplete block designs. Journal of the American Statistical Association 42: 541-561.

Rao C.R. (1956): On the recovery of inter-block information in varietal trials. Sankhy-a 17: 105-114.

Rao C.R. (1959): Expected values of mean squares in the analysis of incomplete block experiments and some comments based on them. Sankhy-a 21: 327336.

Rao C.R. (1971): Unified theory of linear estimation. Sankhy-a, Series A 33: 371-394.

Rao C.R. (1974): Projectors, generalized inverses and the BLUEs. Journal of the Royal Statistical Society, Series B 36: 442-448.

Rao C.R., Mitra S.K. (1971): Generalized Inverse of Matrices and its Applications. Wiley, New York.

R Core Team (2017): R: A language and environment for statistical computing. R Foundation for Statistical Computing, Vienna, Austria. URL https://www.Rproject.org.

Volaufova J. (2009): Heteroscedastic ANOVA: old p values, new views, Statistical Papers 50: 943-962.

Williams E.R. (1975): Efficiency-balanced designs. Biometrika 62: 686-688.

Yates F. (1936): A new method of arranging variety trials involving a large number of varieties. Journal of Agricultural Science 26: 424-455.

Yates F. (1939): The recovery of inter-block information in variety trials arranged in three-dimensional lattices. Annals of Eugenics 9: 136-156.

Yates F. (1940): The recovery of inter-block information in balanced incomplete block designs. Annals of Eugenics 10: 317-325.

\section{Appendix}

\section{Appendix 1}

For formula (13) one has first to show that $\boldsymbol{X}_{1}^{\prime} \boldsymbol{V}^{-1} \boldsymbol{X}_{1}$ can be taken as a $g$-inverse of $\mathrm{D}\left(\hat{\boldsymbol{\tau}}_{*}\right)$ given in (12), i.e., that the equality

$$
\begin{array}{r}
\left(\boldsymbol{I}_{v}-n^{-1} \mathbf{1}_{v} \boldsymbol{r}^{\prime}\right)\left(\boldsymbol{X}_{1}^{\prime} \boldsymbol{V}^{-1} \boldsymbol{X}_{1}\right)^{-1}\left(\boldsymbol{I}_{v}-n^{-1} \boldsymbol{r} \mathbf{1}_{v}^{\prime}\right) \boldsymbol{X}_{1}^{\prime} \boldsymbol{V}^{-1} \boldsymbol{X}_{1}\left(\boldsymbol{I}_{v}\right. \\
\left.-n^{-1} \mathbf{1}_{v} \boldsymbol{r}^{\prime}\right)\left(\boldsymbol{X}_{1}^{\prime} \boldsymbol{V}^{-1} \boldsymbol{X}_{1}\right)^{-1}\left(\boldsymbol{I}_{v}-n^{-1} \boldsymbol{r} \mathbf{1}_{v}^{\prime}\right) \\
=\left(\boldsymbol{I}_{v}-n^{-1} \mathbf{1}_{v} \boldsymbol{r}^{\prime}\right)\left(\boldsymbol{X}_{1}^{\prime} \boldsymbol{V}^{-1} \boldsymbol{X}_{1}\right)^{-1}\left(\boldsymbol{I}_{v}-n^{-1} \boldsymbol{r} \mathbf{1}_{v}^{\prime}\right)
\end{array}
$$

holds. For this, it is sufficient to consider the equalities

$$
\begin{aligned}
\boldsymbol{X}_{1}^{\prime} \boldsymbol{V}^{-1} \boldsymbol{X}_{1}\left(\boldsymbol{I}_{v}-n^{-1} \mathbf{1}_{v} \boldsymbol{r}^{\prime}\right) & =\left(\boldsymbol{I}_{v}-n^{-1} \boldsymbol{r} \mathbf{1}_{v}^{\prime}\right) \boldsymbol{X}_{1}^{\prime} \boldsymbol{V}^{-1} \boldsymbol{X}_{1} \quad \text { and } \\
\left(\boldsymbol{I}_{v}-n^{-1} \boldsymbol{r} \mathbf{1}_{v}^{\prime}\right)\left(\boldsymbol{I}_{v}-n^{-1} \boldsymbol{r} \mathbf{1}_{v}^{\prime}\right) & =\left(\boldsymbol{I}_{v}-n^{-1} \boldsymbol{r} \mathbf{1}_{v}^{\prime}\right) .
\end{aligned}
$$


The second equality is obvious. To prove the first, one has to use the equalities

$$
\begin{aligned}
\boldsymbol{X}_{1}\left(\boldsymbol{I}_{v}-n^{-1} \mathbf{1}_{v} \boldsymbol{r}^{\prime}\right) & =\left(\boldsymbol{I}_{n}-n^{-1} \mathbf{1}_{n} \mathbf{1}_{n}^{\prime}\right) \boldsymbol{X}_{1} \text { and } \\
\boldsymbol{V}^{-1}\left(\boldsymbol{I}_{n}-n^{-1} \mathbf{1}_{n} \mathbf{1}_{n}^{\prime}\right) & =\left(\boldsymbol{I}_{n}-n^{-1} \mathbf{1}_{n} \mathbf{1}_{n}^{\prime}\right) \boldsymbol{V}^{-1},
\end{aligned}
$$

which can easily be checked remembering that $\boldsymbol{X}_{1} \mathbf{1}_{v}=\mathbf{1}_{n}$ and $\mathbf{1}_{n}^{\prime} \boldsymbol{X}_{1}=\boldsymbol{r}^{\prime}$, and also recalling the properties of the matrices $\phi_{1}, \phi_{2}$ and $\phi_{3}$ in formula (3).

Now, with $\boldsymbol{X}_{1}^{\prime} \boldsymbol{V}^{-1} \boldsymbol{X}_{1}$ as a $g$-inverse of $\mathrm{D}\left(\hat{\boldsymbol{\tau}}_{*}\right)$, the equality (13) follows, which can easily be checked noting that $\left(\boldsymbol{I}_{v}-n^{-1} \mathbf{1}_{v} \boldsymbol{r}^{\prime}\right) \hat{\boldsymbol{\tau}}_{*}=\hat{\boldsymbol{\tau}}_{*}$.

\section{Appendix 2}

For formula (14), note that the sum of squares $\mathrm{SS}_{V}$ can, on account of (8), be written as

$$
\begin{array}{r}
\mathrm{SS}_{V}=\boldsymbol{y}^{\prime} \boldsymbol{V}^{-1} \boldsymbol{X}_{1}\left(\boldsymbol{X}_{1}^{\prime} \boldsymbol{V}^{-1} \boldsymbol{X}_{1}\right)^{-1}\left(\boldsymbol{I}_{v}-n^{-1} \boldsymbol{r} \mathbf{1}_{v}^{\prime}\right) \boldsymbol{X}_{1}^{\prime} \boldsymbol{V}^{-1} \boldsymbol{X}\left(\boldsymbol{I}_{v}\right. \\
\left.-n^{-1} \mathbf{1}_{v} \boldsymbol{r}^{\prime}\right)\left(\boldsymbol{X}_{1}^{\prime} \boldsymbol{V}^{-1} \boldsymbol{X}_{1}\right)^{-1} \boldsymbol{X}_{1}^{\prime} \boldsymbol{V}^{-1} \boldsymbol{y}
\end{array}
$$

which, by the equalities

$$
\begin{aligned}
\boldsymbol{X}_{1}^{\prime} \boldsymbol{V}^{-1} \boldsymbol{X}_{1}\left(\boldsymbol{I}_{v}-n^{-1} \mathbf{1}_{v} \boldsymbol{r}^{\prime}\right)\left(\boldsymbol{X}_{1}^{\prime} \boldsymbol{V}^{-1} \boldsymbol{X}_{1}\right)^{-1} & =\boldsymbol{I}_{v}-n^{-1} \boldsymbol{r} \mathbf{1}_{v}^{\prime} \quad \text { and } \\
\left(\boldsymbol{I}_{v}-n^{-1} \mathbf{1}_{v} \boldsymbol{r}^{\prime}\right)\left(\boldsymbol{X}_{1}^{\prime} \boldsymbol{V}^{-1} \boldsymbol{X}_{1}\right)^{-1} & =\left(\boldsymbol{X}_{1}^{\prime} \boldsymbol{V}^{-1} \boldsymbol{X}_{1}\right)^{-1}\left(\boldsymbol{I}_{v}-n^{-1} \boldsymbol{r} \mathbf{1}_{v}^{\prime}\right)
\end{aligned}
$$

(see Appendix 1), can be reduced to the form in (15). As to the sum of squares $\mathrm{SS}_{R}$, its formula (16) follows directly from (14) on account of (4).

\section{Appendix 3}

For the formulae in (29), note that using the known formula

$$
(\boldsymbol{A}+\boldsymbol{B C D})^{-1}=\boldsymbol{A}^{-1}-\boldsymbol{A}^{-1} \boldsymbol{B}\left(\boldsymbol{C}^{-1}+\boldsymbol{D} \boldsymbol{A}^{-1} \boldsymbol{B}\right)^{-1} \boldsymbol{D} \boldsymbol{A}^{-1}
$$

[see, e.g., (A.2.4f) in Mardia, Kent and Bibby (1979)], one can write, on account of $(29)$,

$\boldsymbol{V}^{-1}=\boldsymbol{V}_{*}^{-1}-\boldsymbol{V}_{*}^{-1} n^{-1} \mathbf{1}_{n} \mathbf{1}_{n}^{\prime}\left[\left(\sigma_{3}^{2}-\sigma_{2}^{2}\right)^{-1} \boldsymbol{I}_{n}+n^{-1} \mathbf{1}_{n} \mathbf{1}_{n}^{\prime} \boldsymbol{V}_{*}^{-1} n^{-1} \mathbf{1}_{n} \mathbf{1}_{n}^{\prime}\right]^{-1} n^{-1} \mathbf{1}_{n} \mathbf{1}_{n}^{\prime} \boldsymbol{V}_{*}^{-1}$,

from which

$$
\begin{aligned}
\boldsymbol{X}_{1}^{\prime} \boldsymbol{V}^{-1} \boldsymbol{X}_{1}= & \boldsymbol{X}_{1}^{\prime} \boldsymbol{V}_{*}^{-1} \boldsymbol{X}_{1}-\boldsymbol{X}_{1}^{\prime} \boldsymbol{V}_{*}^{-1} n^{-1} \mathbf{1}_{n} \mathbf{1}_{n}^{\prime}\left[\left(\sigma_{3}^{2}-\sigma_{2}^{2}\right)^{-1} \boldsymbol{I}_{n}\right. \\
& \left.+n^{-1} \mathbf{1}_{n} \mathbf{1}_{n}^{\prime} \boldsymbol{V}_{*}^{-1} n^{-1} \mathbf{1}_{n} \mathbf{1}_{n}^{\prime}\right]^{-1} n^{-1} \mathbf{1}_{n} \mathbf{1}_{n}^{\prime} \boldsymbol{V}_{*}^{-1} \boldsymbol{X}_{1} \\
= & \boldsymbol{X}_{1}^{\prime} \boldsymbol{V}_{*}^{-1} \boldsymbol{X}_{1}-\boldsymbol{X}_{1}^{\prime} \boldsymbol{V}_{*}^{-1} \boldsymbol{X}_{1} \mathbf{1}_{v} n^{-1} \mathbf{1}_{n}^{\prime}\left[\left(\sigma_{3}^{2}-\sigma_{2}^{2}\right)^{-1} \boldsymbol{I}_{n}\right. \\
& \left.+n^{-1} \mathbf{1}_{n} \mathbf{1}_{v}^{\prime} \boldsymbol{X}_{1}^{\prime} \boldsymbol{V}_{*}^{-1} \boldsymbol{X}_{1} \mathbf{1}_{v} n^{-1} \mathbf{1}_{n}^{\prime}\right]^{-1} n^{-1} \mathbf{1}_{n} \mathbf{1}_{v}^{\prime} \boldsymbol{X}_{1}^{\prime} \boldsymbol{V}_{*}^{-1} \boldsymbol{X}_{1} \\
= & {\left[\left(\boldsymbol{X}_{1}^{\prime} \boldsymbol{V}_{*}^{-1} \boldsymbol{X}_{1}\right)^{-1}+n^{-1} \mathbf{1}_{v} \mathbf{1}_{n}^{\prime}\left(\sigma_{3}^{2}-\sigma_{2}^{2}\right) n^{-1} \mathbf{1}_{n} \mathbf{1}_{v}^{\prime}\right]^{-1} } \\
= & {\left[\left(\boldsymbol{X}_{1}^{\prime} \boldsymbol{V}_{*}^{-1} \boldsymbol{X}_{1}\right)^{-1}+\left(\sigma_{3}^{2}-\sigma_{2}^{2}\right) n^{-1} \mathbf{1}_{v} \mathbf{1}_{v}^{\prime}\right]^{-1} . }
\end{aligned}
$$


Taking the inverse of this, one obtains

$$
\left(\boldsymbol{X}_{1}^{\prime} \boldsymbol{V}^{-1} \boldsymbol{X}_{1}\right)^{-1}=\left(\boldsymbol{X}_{1}^{\prime} \boldsymbol{V}_{*}^{-1} \boldsymbol{X}_{1}\right)^{-1}+\left(\sigma_{3}^{2}-\sigma_{2}^{2}\right) n^{-1} \mathbf{1}_{v} \mathbf{1}_{v}^{\prime},
$$

i.e., the formula (30). From (29) it also follows that

$$
\boldsymbol{X}_{1}^{\prime} \boldsymbol{V}^{-1} \boldsymbol{X}_{1}=\boldsymbol{X}_{1}^{\prime} \boldsymbol{V}_{*}^{-1} \boldsymbol{X}_{1}+\left(\sigma_{3}^{-2}-\sigma_{2}^{-2}\right) n^{-1} \boldsymbol{r} \boldsymbol{r}^{\prime},
$$

due to the relation $\mathbf{1}_{n}^{\prime} \boldsymbol{X}_{1}=\boldsymbol{r}^{\prime}$.

Furthermore, with these results the equality (31) can be proved, proceeding as follows:

$$
\begin{aligned}
\hat{\boldsymbol{\tau}}_{*}= & \left(\boldsymbol{I}_{v}-n^{-1} \mathbf{1}_{v} \boldsymbol{r}^{\prime}\right)\left(\boldsymbol{X}_{1}^{\prime} \boldsymbol{V}^{-1} \boldsymbol{X}_{1}\right)^{-1} \boldsymbol{X}_{1}^{\prime} \boldsymbol{V}^{-1} \boldsymbol{y} \\
= & \left(\boldsymbol{I}_{v}-n^{-1} \mathbf{1}_{v} \boldsymbol{r}^{\prime}\right)\left(\boldsymbol{X}_{1}^{\prime} \boldsymbol{V}_{*}^{-1} \boldsymbol{X}_{1}\right)^{-1} \boldsymbol{X}_{1}^{\prime} \boldsymbol{V}^{-1} \boldsymbol{y} \\
= & \left(\boldsymbol{I}_{v}-n^{-1} \mathbf{1}_{v} \boldsymbol{r}^{\prime}\right)\left(\boldsymbol{I}_{v}-n^{-1} \mathbf{1}_{v} \boldsymbol{r}^{\prime}\right)\left(\boldsymbol{X}_{1}^{\prime} \boldsymbol{V}_{*}^{-1} \boldsymbol{X}_{1}\right)^{-1} \boldsymbol{X}_{1}^{\prime} \boldsymbol{V}^{-1} \boldsymbol{y} \\
= & \left(\boldsymbol{I}_{v}-n^{-1} \mathbf{1}_{v} \boldsymbol{r}^{\prime}\right)\left(\boldsymbol{X}_{1}^{\prime} \boldsymbol{V}_{*}^{-1} \boldsymbol{X}_{1}\right)^{-1}\left(\boldsymbol{I}_{v}-n^{-1} \boldsymbol{r} \mathbf{1}_{v}^{\prime}\right) \boldsymbol{X}_{1}^{\prime} \boldsymbol{V}^{-1} \boldsymbol{y} \\
= & \left(\boldsymbol{I}_{v}-n^{-1} \mathbf{1}_{v} \boldsymbol{r}^{\prime}\right)\left(\boldsymbol{X}_{1}^{\prime} \boldsymbol{V}_{*}^{-1} \boldsymbol{X}_{1}\right)^{-1} \boldsymbol{X}_{1}^{\prime}\left(\boldsymbol{I}_{n}-n^{-1} \mathbf{1}_{n} \mathbf{1}_{n}^{\prime}\right) \boldsymbol{V}^{-1} \boldsymbol{y} \\
= & \left(\boldsymbol{I}_{v}-n^{-1} \mathbf{1}_{v} \boldsymbol{r}^{\prime}\right)\left(\boldsymbol{X}_{1}^{\prime} \boldsymbol{V}_{*}^{-1} \boldsymbol{X}_{1}\right)^{-1} \boldsymbol{X}_{1}^{\prime}\left(\boldsymbol{I}_{v}-n^{-1} \mathbf{1}_{n} \mathbf{1}_{n}^{\prime}\right)\left[\boldsymbol{V}_{*}^{-1}\right. \\
& \left.+\left(\sigma_{3}^{-2}-\sigma_{2}^{-2}\right) n^{-1} \mathbf{1}_{n} \mathbf{1}_{n}^{\prime}\right] \boldsymbol{y} \\
= & \left(\boldsymbol{I}_{v}-n^{-1} \mathbf{1}_{v} \boldsymbol{r}^{\prime}\right)\left(\boldsymbol{X}_{1}^{\prime} \boldsymbol{V}_{*}^{-1} \boldsymbol{X}_{1}\right)^{-1} \boldsymbol{X}_{1}^{\prime} \boldsymbol{V}_{*}^{-1}\left(\boldsymbol{I}_{v}-n^{-1} \mathbf{1}_{n} \mathbf{1}_{n}^{\prime}\right) \boldsymbol{y},
\end{aligned}
$$

because $\left(\boldsymbol{I}_{v}-n^{-1} \boldsymbol{r} \mathbf{1}_{v}^{\prime}\right) \boldsymbol{X}_{1}^{\prime}=\boldsymbol{X}_{1}^{\prime}\left(\boldsymbol{I}_{n}-n^{-1} \mathbf{1}_{n} \mathbf{1}_{n}^{\prime}\right)$ and $\left(\boldsymbol{I}_{n}-n^{-1} \mathbf{1}_{n} \mathbf{1}_{n}^{\prime}\right) \boldsymbol{V}_{*}^{-1}=$ $\boldsymbol{V}_{*}^{-1}\left(\boldsymbol{I}_{v}-n^{-1} \mathbf{1}_{n} \mathbf{1}_{n}^{\prime}\right)$, as can easily be checked.

\section{Appendix 4}

Formulae (33) and (34) are found to be equivalent to formulae (15) and (16) respectively. To prove this, it may be helpful first to note the following equalities, which can easily be checked (see also Appendices 1 and 3):

$$
\begin{aligned}
\left(\boldsymbol{X}_{1}^{\prime} \boldsymbol{V}^{-1} \boldsymbol{X}_{1}\right)^{-1}\left(\boldsymbol{I}_{v}-n^{-1} \boldsymbol{r} \mathbf{1}_{v}^{\prime}\right) & =\left(\boldsymbol{X}_{1}^{\prime} \boldsymbol{V}_{*}^{-1} \boldsymbol{X}_{1}\right)^{-1}\left(\boldsymbol{I}_{v}-n^{-1} \boldsymbol{r} \mathbf{1}_{v}^{\prime}\right), \\
\boldsymbol{X}_{1}\left(\boldsymbol{I}_{v}-n^{-1} \mathbf{1}_{v} \boldsymbol{r}^{\prime}\right) & =\left(\boldsymbol{I}_{n}-n^{-1} \mathbf{1}_{n} \mathbf{1}_{n}^{\prime}\right) \boldsymbol{X}_{1}, \\
\left(\boldsymbol{I}_{v}-n^{-1} \boldsymbol{r} \mathbf{1}_{v}^{\prime}\right) \boldsymbol{X}_{1}^{\prime} & =\boldsymbol{X}_{1}^{\prime}\left(\boldsymbol{I}_{n}-n^{-1} \mathbf{1}_{n} \mathbf{1}_{n}^{\prime}\right), \\
\boldsymbol{V}^{-1}\left(\boldsymbol{I}_{n}-n^{-1} \mathbf{1}_{n} \mathbf{1}_{n}^{\prime}\right) & =\boldsymbol{V}_{*}^{-1}\left(\boldsymbol{I}_{n}-n^{-1} \mathbf{1}_{n} \mathbf{1}_{n}^{\prime}\right), \\
\left(\boldsymbol{I}_{n}-n^{-1} \mathbf{1}_{n} \mathbf{1}_{n}^{\prime}\right) \boldsymbol{V}^{-1} & =\left(\boldsymbol{I}_{n}-n^{-1} \mathbf{1}_{n} \mathbf{1}_{n}^{\prime}\right) \boldsymbol{V}_{*}^{-1} \\
\boldsymbol{V}_{*}^{-1}\left(\boldsymbol{I}_{n}-n^{-1} \mathbf{1}_{n} \mathbf{1}_{n}^{\prime}\right) & =\left(\boldsymbol{I}_{n}-n^{-1} \mathbf{1}_{n} \mathbf{1}_{n}^{\prime}\right) \boldsymbol{V}_{*}^{-1} .
\end{aligned}
$$

With these observations, it is easy to proceed as follows:

$$
\begin{aligned}
\mathrm{SS}_{V} & =\boldsymbol{y}^{\prime} \boldsymbol{V}^{-1} \boldsymbol{X}_{1}\left(\boldsymbol{I}_{v}-n^{-1} \mathbf{1}_{v} \boldsymbol{r}^{\prime}\right)\left(\boldsymbol{X}_{1}^{\prime} \boldsymbol{V}^{-1} \boldsymbol{X}_{1}\right)^{-1}\left(\boldsymbol{I}_{v}-n^{-1} \boldsymbol{r} \mathbf{1}_{v}^{\prime}\right) \boldsymbol{X}_{1}^{\prime} \boldsymbol{V}^{-1} \boldsymbol{y} \\
& =\boldsymbol{y}^{\prime} \boldsymbol{V}^{-1} \boldsymbol{X}_{1}\left(\boldsymbol{I}_{v}-n^{-1} \mathbf{1}_{v} \boldsymbol{r}^{\prime}\right)\left(\boldsymbol{X}_{1}^{\prime} \boldsymbol{V}_{*}^{-1} \boldsymbol{X}_{1}\right)^{-1}\left(\boldsymbol{I}_{v}-n^{-1} \boldsymbol{r} \mathbf{1}_{v}^{\prime}\right) \boldsymbol{X}_{1}^{\prime} \boldsymbol{V}^{-1} \boldsymbol{y} \\
& =\boldsymbol{y}^{\prime} \boldsymbol{V}^{-1}\left(\boldsymbol{I}_{n}-n^{-1} \mathbf{1}_{n} \mathbf{1}_{n}^{\prime}\right) \boldsymbol{X}_{1}\left(\boldsymbol{X}_{1}^{\prime} \boldsymbol{V}_{*}^{-1} \boldsymbol{X}_{1}\right)^{-1} \boldsymbol{X}_{1}^{\prime}\left(\boldsymbol{I}_{n}-n^{-1} \mathbf{1}_{n} \mathbf{1}_{n}^{\prime}\right) \boldsymbol{V}^{-1} \boldsymbol{y} \\
& =\boldsymbol{y}^{\prime}\left(\boldsymbol{I}_{n}-n^{-1} \mathbf{1}_{n} \mathbf{1}_{n}^{\prime}\right) \boldsymbol{V}_{*}^{-1} \boldsymbol{X}_{1}\left(\boldsymbol{X}_{1}^{\prime} \boldsymbol{V}_{*}^{-1} \boldsymbol{X}_{1}\right)^{-1} \boldsymbol{X}_{1}^{\prime} \boldsymbol{V}_{*}^{-1}\left(\boldsymbol{I}_{n}-n^{-1} \mathbf{1}_{n} \mathbf{1}_{n}^{\prime}\right) \boldsymbol{y},
\end{aligned}
$$


which, with $\boldsymbol{y}_{*}=\left(\boldsymbol{I}_{n}-n^{-1} \mathbf{1}_{n} \mathbf{1}_{n}^{\prime}\right) \boldsymbol{y}$, is equivalent to the formula (33).

Now, considering formula (16), it will be helpful first to note (recalling Appendix 1) that

$$
\begin{aligned}
& {\left[\boldsymbol{V}^{-1}-\boldsymbol{V}^{-1} \boldsymbol{X}_{1}\left(\boldsymbol{X}_{1}^{\prime} \boldsymbol{V}^{-1} \boldsymbol{X}_{1}\right)^{-1} \boldsymbol{X}_{1}^{\prime} \boldsymbol{V}^{-1}\right] n^{-1} \mathbf{1}_{n} \mathbf{1}_{n}^{\prime} } \\
= & \boldsymbol{V}^{-1} n^{-1} \mathbf{1}_{n} \mathbf{1}_{n}^{\prime}-\boldsymbol{V}^{-1} \boldsymbol{X}_{1}\left(\boldsymbol{X}_{1}^{\prime} \boldsymbol{V}^{-1} \boldsymbol{X}_{1}\right)^{-1} \boldsymbol{X}_{1}^{\prime} \boldsymbol{V}^{-1} n^{-1} \mathbf{1}_{n} \mathbf{1}_{n}^{\prime} \\
= & \boldsymbol{V}^{-1} n^{-1} \mathbf{1}_{n} \mathbf{1}_{n}^{\prime}-\boldsymbol{V}^{-1} \boldsymbol{X}_{1}\left(\boldsymbol{X}_{1}^{\prime} \boldsymbol{V}^{-1} \boldsymbol{X}_{1}\right)^{-1} \boldsymbol{X}_{1}^{\prime} \boldsymbol{V}^{-1} \boldsymbol{X}_{1} \mathbf{1}_{v} n^{-1} \mathbf{1}_{n}^{\prime} \\
= & \boldsymbol{V}^{-1} n^{-1} \mathbf{1}_{n} \mathbf{1}_{n}^{\prime}-\boldsymbol{V}^{-1} \boldsymbol{X}_{1} \mathbf{1}_{v} n^{-1} \mathbf{1}_{n}^{\prime}=\boldsymbol{V}^{-1} n^{-1} \mathbf{1}_{n} \mathbf{1}_{n}^{\prime}-\boldsymbol{V}^{-1} n^{-1} \mathbf{1}_{n} \mathbf{1}_{n}^{\prime}=\mathbf{O} .
\end{aligned}
$$

With this result formula (16) can be written as

$$
\begin{aligned}
& \mathrm{SS}_{R}=\boldsymbol{y}^{\prime}\left[\boldsymbol{V}^{-1}-\boldsymbol{V}^{-1} \boldsymbol{X}_{1}\left(\boldsymbol{X}_{1}^{\prime} \boldsymbol{V}^{-1} \boldsymbol{X}_{1}\right)^{-1} \boldsymbol{X}_{1}^{\prime} \boldsymbol{V}^{-1}\right] \boldsymbol{y} \\
& =\boldsymbol{y}^{\prime}\left[\boldsymbol{V}^{-1}-\boldsymbol{V}^{-1} \boldsymbol{X}_{1}\left(\boldsymbol{X}_{1}^{\prime} \boldsymbol{V}^{-1} \boldsymbol{X}_{1}\right)^{-1} \boldsymbol{X}_{1}^{\prime} \boldsymbol{V}^{-1}\right]\left(\boldsymbol{I}_{n}-n^{-1} \mathbf{1}_{n} \mathbf{1}_{n}^{\prime}\right) \boldsymbol{y} \\
& =\boldsymbol{y}^{\prime}\left[\boldsymbol{V}^{-1}-\boldsymbol{V}^{-1} \boldsymbol{X}_{1}\left(\boldsymbol{X}_{1}^{\prime} \boldsymbol{V}^{-1} \boldsymbol{X}_{1}\right)^{-1} \boldsymbol{X}_{1}^{\prime} \boldsymbol{V}^{-1}\right]\left(\boldsymbol{I}_{n}-n^{-1} \mathbf{1}_{n} \mathbf{1}_{n}^{\prime}\right)\left(\boldsymbol{I}_{n}-n^{-1} \mathbf{1}_{n} \mathbf{1}_{n}^{\prime}\right) \boldsymbol{y} \\
& =\boldsymbol{y}^{\prime}\left[\boldsymbol{V}^{-1}\left(\boldsymbol{I}_{n}-n^{-1} \mathbf{1}_{n} \mathbf{1}_{n}^{\prime}\right)-\boldsymbol{V}^{-1} \boldsymbol{X}_{1}\left(\boldsymbol{X}_{1}^{\prime} \boldsymbol{V}^{-1} \boldsymbol{X}_{1}\right)^{-1} \boldsymbol{X}_{1}^{\prime} \boldsymbol{V}^{-1}\left(\boldsymbol{I}_{n}-n^{-1} \mathbf{1}_{n} \mathbf{1}_{n}^{\prime}\right)\right] \boldsymbol{y}_{*} \\
& =\boldsymbol{y}^{\prime}\left[\left(\boldsymbol{I}_{n}-n^{-1} \mathbf{1}_{n} \mathbf{1}_{n}^{\prime}\right) \boldsymbol{V}^{-1}-\boldsymbol{V}^{-1} \boldsymbol{X}_{1}\left(\boldsymbol{X}_{1}^{\prime} \boldsymbol{V}^{-1} \boldsymbol{X}_{1}\right)^{-1} \boldsymbol{X}_{1}^{\prime}\left(\boldsymbol{I}_{n}-n^{-1} \mathbf{1}_{n} \mathbf{1}_{n}^{\prime}\right) \boldsymbol{V}^{-1}\right] \boldsymbol{y}_{*} \\
& =\boldsymbol{y}^{\prime}\left[\left(\boldsymbol{I}_{n}-n^{-1} \mathbf{1}_{n} \mathbf{1}_{n}^{\prime}\right) \boldsymbol{V}_{*}^{-1}-\boldsymbol{V}^{-1} \boldsymbol{X}_{1}\left(\boldsymbol{X}_{1}^{\prime} \boldsymbol{V}^{-1} \boldsymbol{X}_{1}\right)^{-1} \boldsymbol{X}_{1}^{\prime}\left(\boldsymbol{I}_{n}-n^{-1} \mathbf{1}_{n} \mathbf{1}_{n}^{\prime}\right) \boldsymbol{V}_{*}^{-1}\right] \boldsymbol{y}_{*} \\
& =\boldsymbol{y}^{\prime}\left[\left(\boldsymbol{I}_{n}-n^{-1} \mathbf{1}_{n} \mathbf{1}_{n}^{\prime}\right) \boldsymbol{V}_{*}^{-1}-\boldsymbol{V}^{-1} \boldsymbol{X}_{1}\left(\boldsymbol{X}_{1}^{\prime} \boldsymbol{V}^{-1} \boldsymbol{X}_{1}\right)^{-1}\left(\boldsymbol{I}_{v}-n^{-1} \boldsymbol{r} \mathbf{1}_{v}^{\prime}\right) \boldsymbol{X}_{1}^{\prime} \boldsymbol{V}_{*}^{-1}\right] \boldsymbol{y}_{*} \\
& =\boldsymbol{y}^{\prime}\left[\left(\boldsymbol{I}_{n}-n^{-1} \mathbf{1}_{n} \mathbf{1}_{n}^{\prime}\right) \boldsymbol{V}_{*}^{-1}-\boldsymbol{V}^{-1} \boldsymbol{X}_{1}\left(\boldsymbol{X}_{1}^{\prime} \boldsymbol{V}_{*}^{-1} \boldsymbol{X}_{1}\right)^{-1}\left(\boldsymbol{I}_{v}-n^{-1} \boldsymbol{r} \mathbf{1}_{v}^{\prime}\right) \boldsymbol{X}_{1}^{\prime} \boldsymbol{V}_{*}^{-1}\right] \boldsymbol{y}_{*} \\
& =\boldsymbol{y}^{\prime}\left[\left(\boldsymbol{I}_{n}-n^{-1} \mathbf{1}_{n} \mathbf{1}_{n}^{\prime}\right) \boldsymbol{V}_{*}^{-1}-\boldsymbol{V}^{-1} \boldsymbol{X}_{1}\left(\boldsymbol{I}_{v}-n^{-1} \mathbf{1}_{v} \boldsymbol{r}^{\prime}\right)\left(\boldsymbol{X}_{1}^{\prime} \boldsymbol{V}_{*}^{-1} \boldsymbol{X}_{1}\right)^{-1} \boldsymbol{X}_{1}^{\prime} \boldsymbol{V}_{*}^{-1}\right] \boldsymbol{y}_{*} \\
& =\boldsymbol{y}^{\prime}\left[\left(\boldsymbol{I}_{n}-n^{-1} \mathbf{1}_{n} \mathbf{1}_{n}^{\prime}\right) \boldsymbol{V}_{*}^{-1}-\boldsymbol{V}^{-1}\left(\boldsymbol{I}_{n}-n^{-1} \mathbf{1}_{n} \mathbf{1}_{n}^{\prime}\right) \boldsymbol{X}_{1}\left(\boldsymbol{X}_{1}^{\prime} \boldsymbol{V}_{*}^{-1} \boldsymbol{X}_{1}\right)^{-1} \boldsymbol{X}_{1}^{\prime} \boldsymbol{V}_{*}^{-1}\right] \boldsymbol{y}_{*} \\
& =\boldsymbol{y}^{\prime}\left[\left(\boldsymbol{I}_{n}-n^{-1} \mathbf{1}_{n} \mathbf{1}_{n}^{\prime}\right) \boldsymbol{V}_{*}^{-1}-\left(\boldsymbol{I}_{n}-n^{-1} \mathbf{1}_{n} \mathbf{1}_{n}^{\prime}\right) \boldsymbol{V}_{*}^{-1} \boldsymbol{X}_{1}\left(\boldsymbol{X}_{1}^{\prime} \boldsymbol{V}_{*}^{-1} \boldsymbol{X}_{1}\right)^{-1} \boldsymbol{X}_{1}^{\prime} \boldsymbol{V}_{*}^{-1}\right] \boldsymbol{y}_{*} \\
& =\boldsymbol{y}_{*}^{\prime}\left[\boldsymbol{V}_{*}^{-1}-\boldsymbol{V}_{*}^{-1} \boldsymbol{X}_{1}\left(\boldsymbol{X}_{1}^{\prime} \boldsymbol{V}_{*}^{-1} \boldsymbol{X}_{1}\right)^{-1} \boldsymbol{X}_{1}^{\prime} \boldsymbol{V}_{*}^{-1}\right] \boldsymbol{y}_{*},
\end{aligned}
$$

which, with $\boldsymbol{y}_{*}=\left(\boldsymbol{I}_{n}-n^{-1} \mathbf{1}_{n} \mathbf{1}_{n}^{\prime}\right) \boldsymbol{y}$, is equivalent to (34).

\section{Appendix 5}

For formula (41), note that, from (40),

$$
\begin{aligned}
\operatorname{SS}\left(\boldsymbol{U}_{\mathrm{A}}\right)+\operatorname{SS}\left(\boldsymbol{U}_{\mathrm{B}}\right)= & \hat{\boldsymbol{\tau}}_{*}^{\prime}\left\{\boldsymbol{U}_{\mathrm{A}}\left[\boldsymbol{U}_{\mathrm{A}}^{\prime}\left(\boldsymbol{X}_{1}^{\prime} \boldsymbol{V}_{*}^{-1} \boldsymbol{X}_{1}\right)^{-1} \boldsymbol{U}_{\mathrm{A}}\right]^{-} \boldsymbol{U}_{\mathrm{A}}^{\prime}\right. \\
& \left.+\boldsymbol{U}_{\mathrm{B}}\left[\boldsymbol{U}_{\mathrm{B}}^{\prime}\left(\boldsymbol{X}_{1}^{\prime} \boldsymbol{V}_{*}^{-1} \boldsymbol{X}_{1}\right)^{-1} \boldsymbol{U}_{\mathrm{B}}\right]^{-} \boldsymbol{U}_{\mathrm{B}}^{\prime}\right\} \hat{\boldsymbol{\tau}}_{*} \\
= & \hat{\boldsymbol{\tau}}^{\prime}\left(\boldsymbol{I}_{v}-n^{-1} \boldsymbol{r} \mathbf{1}_{v}^{\prime}\right)\left\{\boldsymbol{U}_{\mathrm{A}}\left[\boldsymbol{U}_{\mathrm{A}}^{\prime}\left(\boldsymbol{X}_{1}^{\prime} \boldsymbol{V}_{*}^{-1} \boldsymbol{X}_{1}\right)^{-1} \boldsymbol{U}_{\mathrm{A}}\right]^{-} \boldsymbol{U}_{\mathrm{A}}^{\prime}\right. \\
& \left.+\boldsymbol{U}_{\mathrm{B}}\left[\boldsymbol{U}_{\mathrm{B}}^{\prime}\left(\boldsymbol{X}_{1}^{\prime} \boldsymbol{V}_{*}^{-1} \boldsymbol{X}_{1}\right)^{-1} \boldsymbol{U}_{\mathrm{B}}\right]^{-} \boldsymbol{U}_{\mathrm{B}}^{\prime}\right\}\left(\boldsymbol{I}_{v}-n^{-1} \mathbf{1}_{v} \boldsymbol{r}^{\prime}\right) \hat{\boldsymbol{\tau}},
\end{aligned}
$$

which, because $U_{\mathrm{A}}^{\prime} \mathbf{1}_{v}=\mathbf{0}=\boldsymbol{U}_{\mathrm{B}}^{\prime} \mathbf{1}_{v}$, reduces to

$$
\begin{aligned}
\operatorname{SS}\left(\boldsymbol{U}_{\mathrm{A}}\right)+\mathrm{SS}\left(\boldsymbol{U}_{\mathrm{B}}\right)= & \hat{\boldsymbol{\tau}}^{\prime}\left\{\boldsymbol{U}_{\mathrm{A}}\left[\boldsymbol{U}_{\mathrm{A}}^{\prime}\left(\boldsymbol{X}_{1}^{\prime} \boldsymbol{V}_{*}^{-1} \boldsymbol{X}_{1}\right)^{-1} \boldsymbol{U}_{\mathrm{A}}\right]^{-} \boldsymbol{U}_{\mathrm{A}}^{\prime}\right. \\
& \left.+\boldsymbol{U}_{\mathrm{B}}\left[\boldsymbol{U}_{\mathrm{B}}^{\prime}\left(\boldsymbol{X}_{1}^{\prime} \boldsymbol{V}_{*}^{-1} \boldsymbol{X}_{1}\right)^{-1} \boldsymbol{U}_{\mathrm{B}}\right]^{-} \boldsymbol{U}_{\mathrm{B}}^{\prime}\right\} \hat{\boldsymbol{\tau}} .
\end{aligned}
$$

On the other hand, from (14) and Appendix 3,

$$
\begin{aligned}
\mathrm{SS}_{V}=\hat{\boldsymbol{\tau}}_{*}^{\prime} \boldsymbol{X}_{1}^{\prime} \boldsymbol{V}^{-1} \boldsymbol{X}_{1} \hat{\boldsymbol{\tau}}_{*} & =\hat{\boldsymbol{\tau}}^{\prime}\left(\boldsymbol{I}_{v}-n^{-1} \boldsymbol{r} \mathbf{1}_{v}^{\prime}\right) \boldsymbol{X}_{1}^{\prime} \boldsymbol{V}^{-1} \boldsymbol{X}_{1}\left(\boldsymbol{I}_{v}-n^{-1} \mathbf{1}_{v} \boldsymbol{r}^{\prime}\right) \hat{\boldsymbol{\tau}} \\
& =\hat{\boldsymbol{\tau}}^{\prime}\left(\boldsymbol{I}_{v}-n^{-1} \boldsymbol{r} \mathbf{1}_{v}^{\prime}\right) \boldsymbol{X}_{1}^{\prime} \boldsymbol{V}_{*}^{-1} \boldsymbol{X}_{1}\left(\boldsymbol{I}_{v}-n^{-1} \mathbf{1}_{v} \boldsymbol{r}^{\prime}\right) \hat{\boldsymbol{\tau}}
\end{aligned}
$$


Hence, for any $\hat{\boldsymbol{\tau}}$, the equality (41) holds if and only if

$$
\begin{aligned}
\boldsymbol{U}_{\mathrm{A}}\left[\boldsymbol{U}_{\mathrm{A}}^{\prime}\left(\boldsymbol{X}_{1}^{\prime} \boldsymbol{V}_{*}^{-1} \boldsymbol{X}_{1}\right)^{-1} \boldsymbol{U}_{\mathrm{A}}\right]^{-} \boldsymbol{U}_{\mathrm{A}}^{\prime} & +\boldsymbol{U}_{\mathrm{B}}\left[\boldsymbol{U}_{\mathrm{B}}^{\prime}\left(\boldsymbol{X}_{1}^{\prime} \boldsymbol{V}_{*}^{-1} \boldsymbol{X}_{1}\right)^{-1} \boldsymbol{U}_{\mathrm{B}}\right]^{-} \boldsymbol{U}_{\mathrm{B}}^{\prime} \\
& =\left(\boldsymbol{I}_{v}-n^{-1} \boldsymbol{r} \mathbf{1}_{v}^{\prime}\right) \boldsymbol{X}_{1}^{\prime} \boldsymbol{V}_{*}^{-1} \boldsymbol{X}_{1}\left(\boldsymbol{I}_{v}-n^{-1} \mathbf{1}_{v} \boldsymbol{r}^{\prime}\right) \\
& =\boldsymbol{X}_{1}^{\prime} \boldsymbol{V}_{*}^{-1} \boldsymbol{X}_{1}\left(\boldsymbol{I}_{v}-n^{-1} \mathbf{1}_{v} \boldsymbol{r}^{\prime}\right),
\end{aligned}
$$

because $\left(\boldsymbol{I}_{v}-n^{-1} \boldsymbol{r} \mathbf{1}_{v}^{\prime}\right) \boldsymbol{X}_{1}^{\prime} \boldsymbol{V}_{*}^{-1} \boldsymbol{X}_{1}=\boldsymbol{X}_{1}^{\prime} \boldsymbol{V}_{*}^{-1} \boldsymbol{X}_{1}\left(\boldsymbol{I}_{v}-n^{-1} \mathbf{1}_{v} \boldsymbol{r}^{\prime}\right)$. Now, premultiplying by $\left(\boldsymbol{X}_{1}^{\prime} \boldsymbol{V}_{*}^{-1} \boldsymbol{X}_{1}\right)^{-1}$, one obtains the condition (42). 